\title{
Single-Step Chemical Vapor Deposition Growth of
}

\section{Platinum Nanocrystal: Monolayer $\mathrm{MoS}_{2}$ Dendrite}

\section{Hybrid Materials for Efficient Electrocatalysis}

Wenshuo Xu ${ }^{1,2}$, Sha Li $^{1}$, Gyeong Hee Ryu ${ }^{1}$, Peng Tang ${ }^{1}$, Mauro Pasta ${ }^{1,2}$, Jamie H. Warner ${ }^{3,4,5 *}$

${ }^{1}$ Department of Materials, University of Oxford, Parks Road, Oxford, OX1 3PH, United

\section{Kingdom}

${ }^{2}$ Oxford Suzhou Centre for Advanced Research, 388 Ruoshui Road, Suzhou 215123, Jiangsu

\section{Province, P. R. China}

${ }^{3}$ Walker Department of Mechanical Engineering, The University of Texas at Austin, 204 East

Dean Keeton Street, Austin, Texas, 78712, United States

${ }^{4}$ Materials Graduate Program, Texas Materials Institute, The University of Texas at Austin, 204

East Dean Keeton Street, Austin, Texas, 78712, United States

${ }^{5}$ Department of Electrical and Computer Engineering, The University of Texas at Austin, 2501

Speedway, Austin Texas, 78712, United States

*jamie.warner@austin.utexas.edu;

\begin{abstract}
Two-dimensional (2D) molybdenum disulfide $\left(\mathrm{MoS}_{2}\right)$ has excellent electrocatalytic behavior for the hydrogen evolution reaction (HER), where the catalysis of $2 \mathrm{H}$ phase originates from its edges, defects, and strains. Most synthetic methods to activate the electrochemically inert basal planes with catalytic active metals are completed by sequential steps. However, this is extremely time-
\end{abstract}


consuming and lacks production scalability. Herein, we develop a one-step strategy to achieve efficient electrocatalyst of $\mathrm{Pt}: \mathrm{MoS}_{2}$ hybrid utilizing atmospheric pressure chemical vapor deposition synthesis on a conductive glassy carbon (GC) plate that can be directly employed as the working electrode in the HER. The monolayer thickness ensures decreased interlayer electron hopping and increased efficiency of the charge transfer from the electrode. We tune the domain morphology by controlling the precursor flux to enter kinetic or thermodynamic growth regime, delivering dendritic or triangular shape. The materials chemistry undertaken provides fundamental insights into the instability of $\mathrm{Pt}$ as metal substitutional dopants in the $\mathrm{MoS}_{2}$ lattice, and instead the stable configuration observed is with Pt as highly dispersed small nanocrystals and single atoms bound to the $\mathrm{MoS}_{2}$ surface. The Pt functionalization at a reduced loading level modulates the favorable HER pathway and triggers synergies in the cocatalyst, which exhibits onset potential of $48 \mathrm{mV}$, Tafel slope of $46 \mathrm{mV} \mathrm{dec}{ }^{-1}$, and exchange current density of $110 \mu \mathrm{A} \mathrm{cm}^{-2}$. The enriched edges and defects of dendrite endow it superiority to the triangle, with regard to the density of catalytic sites, synergistic effects, along with electrical resistance. These underpin the positive role of large dendritic $\mathrm{MoS}_{2}$ monolayer as Pt scaffold in water electrolysis.

\section{INTRODUCTION}

Efficient hydrogen evolution via water splitting is an eco-friendly way to generate clean energy. ${ }^{1,2}$ This is typically achieved by involving electrocatalysts to reduce the overpotential of the reaction. ${ }^{1}$ Platinum on carbon $(\mathrm{Pt} / \mathrm{C})$ displays the highest catalytic activity for the HER with a near-zero overpotential, but its use has been subject to the scarcity of natural resources. ${ }^{3}$ Given the good accessibility and outstanding electrocatalytic properties of $\mathrm{MoS}_{2}$, it has been considered as a promising candidate to replace the costly Pt metal. ${ }^{4}$ The exposed edges of $\mathrm{MoS}_{2}$ have been proven 
to be electrocatalytically active for $\mathrm{HER}^{5},{ }^{5}$ attracting intense efforts on the design of its morphology. ${ }^{6-11}$ Till now, enhanced HER performance has been realized by a variety of $\mathrm{MoS}_{2}$ nanostructures rich in edges, such as porous frameworks,,${ }^{6,7}$ vertically aligned atomic layers, ${ }^{8}$ randomly oriented nanoflakes, ${ }^{9}$ and so on.

Chemical vapor deposition (CVD) has been extensively adopted to yield large-scale twodimensional (2D) materials for industrial uptake. Nonetheless, distinct from the edges, the semiconducting basal plane of the $2 \mathrm{H}$-phase $\mathrm{MoS}_{2}$ prepared by CVD is electrochemically inert, rendering it inferior to the metallic 1T-phase $\mathrm{MoS}_{2}$ in the HER, for which the synthesis approach (e.g., solution-based lithium intercalation) is unscalable. ${ }^{12-14}$ Taking these into consideration, diverse approaches have been attempted to activate the basal plane of atomically thin $\mathrm{MoS}_{2}$ grown by CVD, which include defect engineering, ${ }^{15,16}$ metal doping, ${ }^{17}$ strain applying, ${ }^{15}$ field tuning, ${ }^{18}$ and $\mathrm{MoS}_{2}-$ substrate interaction optimizing. ${ }^{19}$ It has also been corroborated that hybridizing $\mathrm{MoS}_{2}$ with other materials can accelerate the electron transport and/or introduce synergies, therefore enhancing the HER kinetics. ${ }^{20-24}$ These can improve the catalysts' efficiency, but have at least one of the following drawbacks: (1) complicated preparations are often required prior to the applications; ${ }^{25}(2)$ some physical properties of $\mathrm{MoS}_{2}$ are sacrificed due to the damage to its crystal lattice, ${ }^{15,16}$ (3) the HER efficiency of such noble metal-free catalysts discovered so far are generally poorer than those comprising $\mathrm{Pt}^{26,27}$ In terms of the hybrid nanocomposites, the microstructure and dispersion of the particles along with their interaction with the support deserve extra attention considering the possibility of agglomeration, which would decrease the hybrid's electrocatalytic activities and durability. ${ }^{27,28}$ Earlier report has elucidated that introducing single heteroatoms and metal clusters in $\mathrm{MoS}_{2}$ can modify its electronic structure, whereby bringing excellent catalytic performances. ${ }^{29}$ Thus, an inclusion of $\mathrm{Pt}$ is still favored to yield highly efficient $\mathrm{MoS}_{2}$ 
electrocatalysts, and the structural optimization of Pt would be crucial to minimize the needed mass. For this, one can downsize the loaded Pt species and facilitate their dispersion across a certain support, so as to increase the electrochemical active surface area and obstruct the nanoparticles from sintering, thereby improving the catalyst's activity and durability. The microstructure of the Pt nanocrystals atop pre-grown $\mathrm{MoS}_{2}$ monolayer has been shown, ${ }^{7,30}$ yet doping $2 \mathrm{D}$ crystals down to the nanoscale during their CVD synthesis has remained challenging, especially while building a scaffold rich with catalytic sites at the same time. More importantly, the interactions between transition metal dichalcogenides (TMDs) and noble metal dopants along with the HER mechanism of the as-attained electrocatalysts have not been unveiled thoroughly.

In this work, we explore the direct hybridization of large and stable Pt:MoS 2 dendrite cocatalysts on an HER electrode using a scalable synthetic technique. The growth is controlled to prevent the formation of Pt:Mo alloys, where the Mo atoms are electrochemically inert. Our strategy delivers dendritic shape and monolayer nature of $\mathrm{MoS}_{2}$, offering high densities of catalytically active centers (i.e., its edges and defects) and maximized charge transfer. The Pt species are stabilized on the $\mathrm{MoS}_{2}$ as single atoms and nanosized clusters, displaying large specific surface area at low loading. The decorated Pt activates the basal plane of $\mathrm{MoS}_{2}$, contributing to enhanced HER activities in conjunction with the edges and defects of $\mathrm{MoS}_{2}$ and arising synergetic behaviors. The domain morphology, crystal structure, chemical states, and elemental composition are studied by scanning electron microscopy (SEM), annular dark field scanning transmission electron microscopy (ADF-STEM), X-ray photoelectron spectroscopy (XPS), and energy dispersive X-ray (EDX) analysis, respectively. The electrocatalytic performance is investigated in acidic media by linear sweep voltammetry (LSV) and cycling tests. The electrocatalytic behavior 
of this special hybrid system is probed, which demonstrates promise as an advanced catalyst for HER.

\section{RESULTS AND DISCUSSION}

Monolayer $\mathrm{MoS}_{2}$ has been grown by atmospheric pressure chemical vapor deposition (APCVD), which is a scalable method to produce high-quality $2 \mathrm{D}$ materials in a controllable manner, without supplemental requirement of vacuum apparatus. Since the highly conductive glassy carbon (GC) exhibiting negligible catalysis is widely used as the working electrode in the HER, it is worth figuring out the synthesis conditions of efficient nanocatalyst on a GC thin plate. This can help to exempt any follow-up procedures such as mechanical transfer and surface cleaning treatment, which always take time plus efforts and deteriorate the properties of the catalyst. Figure 1a illustrates the sample preparation procedures and Figure $1 \mathrm{~b}$ depicts the CVD setup utilized to grow Pt:MoS ${ }_{2}$ hybrids over a large area. We have selected acetone as the solvent for the hexachloroplatinic acid $\left(\mathrm{H}_{2} \mathrm{PtCl}_{6}\right) \mathrm{Pt}$ source, because of its low cost, wide commercial availability, and low boiling point thus enabling a single-step synthesis. The solution is first drop-casted and applied evenly onto the GC surface, then the $\mathrm{H}_{2} \mathrm{PtCl}_{6}$ is reduced into $\mathrm{Pt}$ metal by the hydrogen $\left(\mathrm{H}_{2}\right)$ gas transported with argon (Ar) through the CVD system. After that, the Pt:MoS 2 dendrites are produced via four stages, as presented in Figure 1c. (1) Nucleation. The formation of $\mathrm{MoS}_{2}$ nuclei is preferentially initiated around the Pt, attributed to the lower energy barrier at these heterogeneous sites. The introduced $\mathrm{H}_{2}$ reduces the $\mathrm{H}_{2} \mathrm{PtCl}_{6}$ to $\mathrm{Pt}$ and might also play a role in the defect engineering of $\mathrm{MoS}_{2}$ by generating numerous sulfur (S) vacancies, grain boundaries, as well as micro-cracks/holes in the $\mathrm{MoS}_{2}$ basal plane. These undercoordinated sites are not only catalytically active for the HER, ${ }^{14-16,25,31}$ but also display high binding affinity of the doped 
Pt. ${ }^{7,30,32,33}$ (2) Main growth. Aiming to yield large dendrites, we adjust the growth from thermodynamic mode to kinetic mode via precise control over the relative concentration of the precursors, which is technically realizable by placing the GC support in vicinity of the tube wall. Specific diffusion-limited aggregation (DLA) is driven by a high excess of S vapor compared with the Mo vapor on the substrate surface. In such process, the mass transport coefficient of the gaseous precursors is considerably larger than the reaction rate constant $\left(h_{\mathrm{g}} \gg k_{\mathrm{s}}\right)$. Consequently, with the diffusion of the reactants being the rate-determining step, highly dendritic structures are produced with small fractal dimension and large domain size. ${ }^{10,34}$ To ensure a consistently laminar rather than turbulent flow and permit the growth of uniform monolayer domains, the Reynold's number has been maintained below $200(\operatorname{Re}<200)$ in the reaction system by controlling the total velocity of the carrier gas flow. (3) Edge attachment. We include this stage to avoid the stacking, given that decreasing the layer number can increase the hopping of electrons from the electrode onto the hybrid surface, whereby facilitating the electrocatalytic activities. ${ }^{35}$ The atoms residing on the first grown layer migrate freely and attach to the nearest domain edges, instead of being bound on the surface and forming adlayers. This helps to realize higher thickness uniformity and larger domain size. (4) Thermal annealing, which has been designed to increase the hybrid's stability by enhancing the physisorption at the $\mathrm{Pt}-\mathrm{MoS}_{2}$ interface, which will be discussed in more detail in the electrochemistry section. 


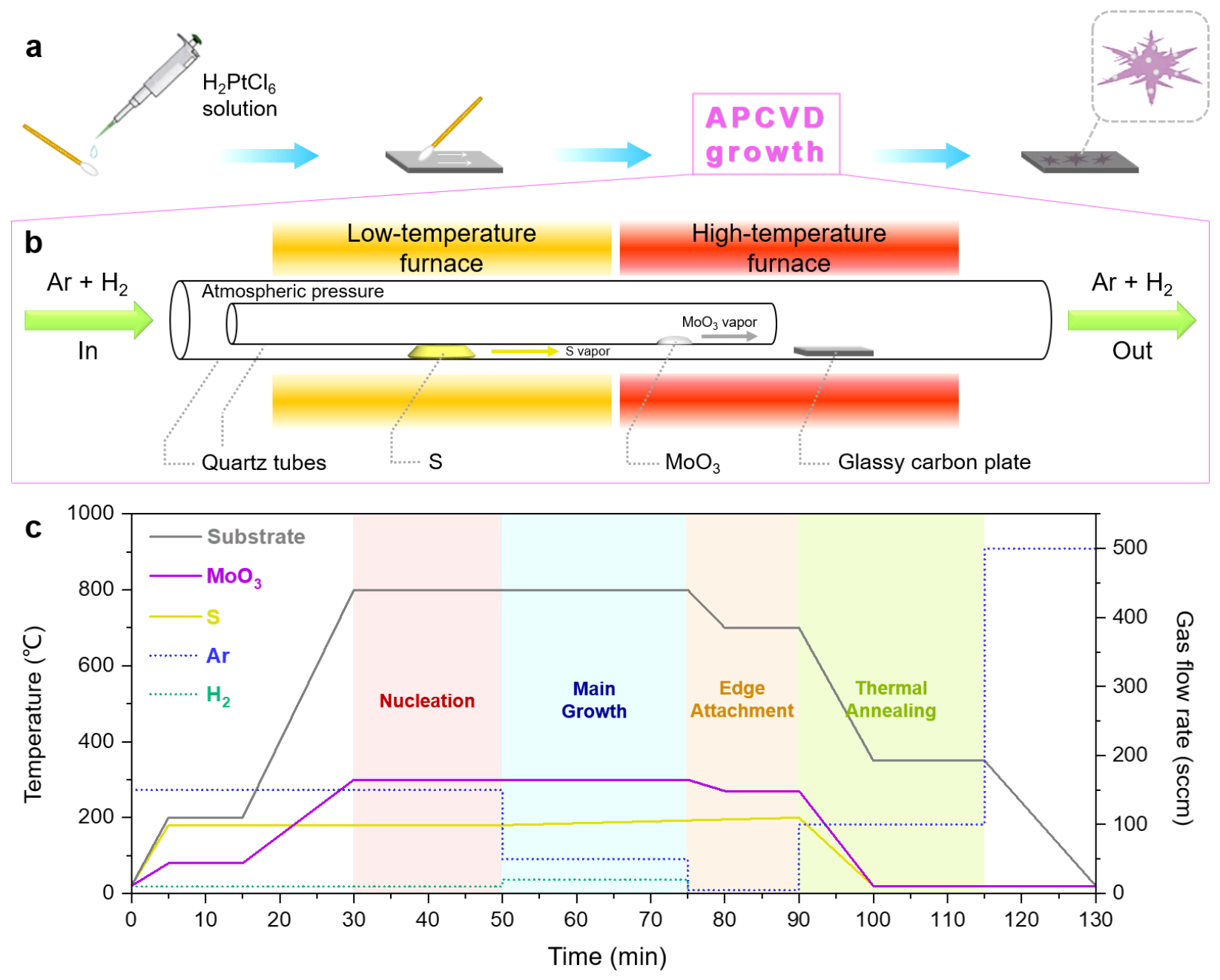

Figure 1. Production of the Pt: $\mathrm{MoS}_{2}$ hybrids. (a) Procedures: drop-casting of $\mathrm{H}_{2} \mathrm{PtCl}_{6}$ acetone solution with a $\mu \mathrm{L}$ burette onto GC plate surface, application of the solution with cotton tip, APCVD growth, and an asprepared sample with the silver balls representing the decorated Pt. (b) Schematic diagram of the APCVD reaction system. (c) Programmed temperature and carrier gas flow rate as a function of the time for APCVD.

Figure 2a shows the APCVD-grown Pt:MoS 2 monolayer dendrites, whose large sizes allow high capacity of Pt. The six-fold symmetric backbones of the dendritic $\mathrm{MoS}_{2}$ domains are also observed for those produced on $\mathrm{SiO}_{2} / \mathrm{Si}$ support. ${ }^{10}$ Originating from the cyclic defects in $\mathrm{MoS}_{2}$ nuclei, ${ }^{36}$ these six directions are associated with the hexagonal symmetry of the $\mathrm{MoS}_{2}$ crystal structure and the three axis of zigzag lattice directions that have different growth rates compared to the arm- 
chair directions, and are therefore independent of the growth substrate. The domain thickness has been measured by atomic force microscope (AFM) measurements (Figure 2c-e), and the values of $\sim 8-10 \AA$ are in good agreement with previous studies for monolayer $\mathrm{MoS}_{2} \cdot{ }^{37,38}$ Figure $2 \mathrm{c}$ shows that $\mathrm{Pt}$ tends to decorate the edges and grain boundaries of the dendritic $\mathrm{MoS}_{2}$ when the doping level is low, whereas these do not happen for pristine $\mathrm{MoS}_{2}$ dendrite (Figure S1), proving that the observed features are from the Pt but not $\mathrm{MoS}_{x}$ clusters aggregated during the CVD growth. The edges and grain boundaries contain larger numbers of dangling bonds that have a higher affinity for absorbing Pt atoms, which could easily grow/aggregate into Pt nanocrystals/nanoclusters owing to their high mobility across monolayer $\mathrm{MoS}_{2} .^{7,33}$ The SEM image of the triangular-shaped Pt:MoS 2 hybrids are displayed in Figure S2, where the Pt nanoclusters are deposited on both the GC substrate and the $\mathrm{MoS}_{2}$ surfaces. At heavy Pt loading >5 wt \%, however, a stable intermetallic Pt:Mo compound is formed along the Pt-Mo phase diagram. ${ }^{39}$ As a result, no defined 2D structure but small isolated bulky crystals are grown (Figure S3). 

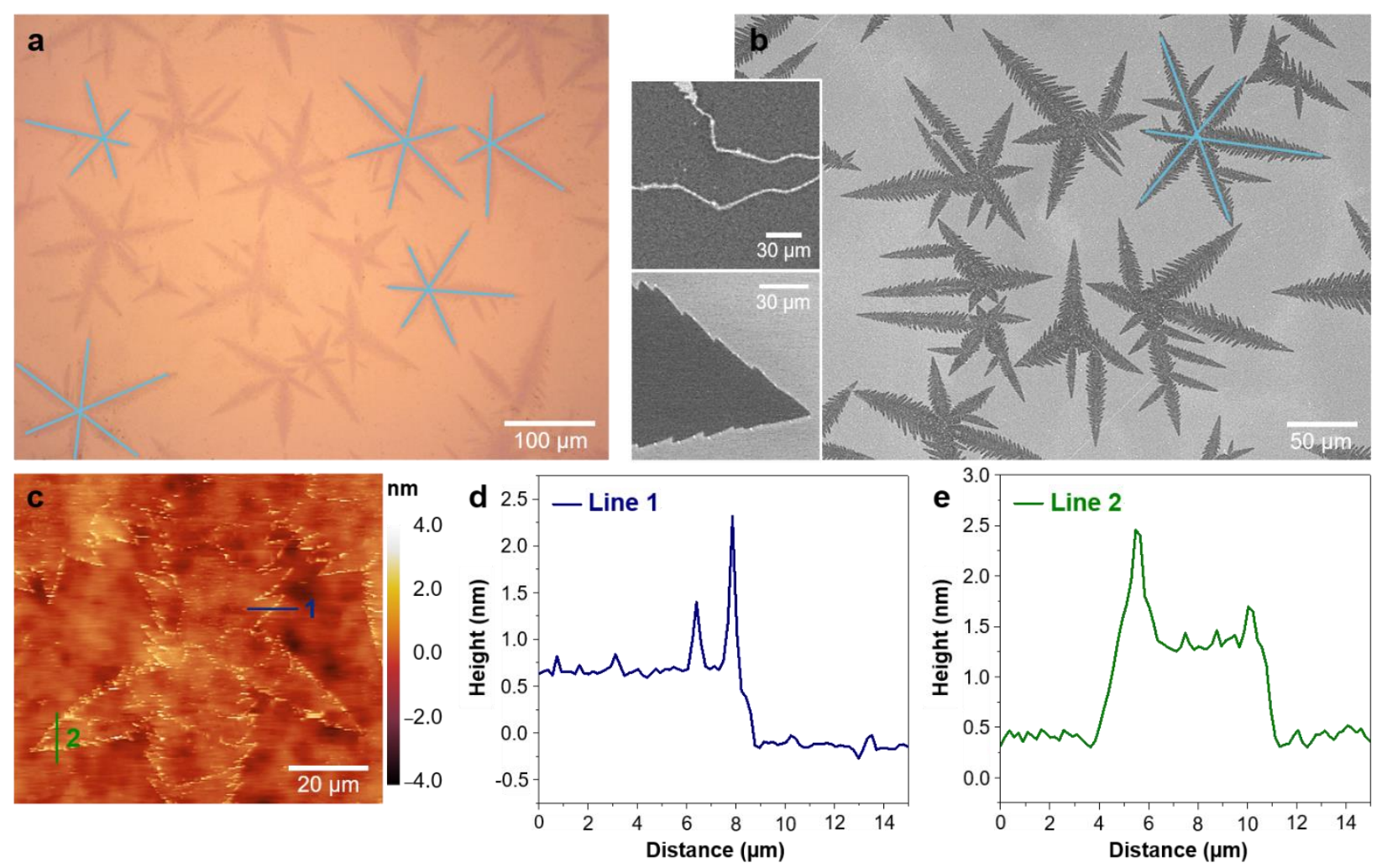

Figure 2. (a) Optical microscopic image and (b) SEM image of the APCVD-grown monolayer $\mathrm{MoS}_{2}$ dendrites. Insets: magnified SEM images showing the Pt nanoparticles at the grain boundaries (upper panel) and the edges (lower panel). (c) AFM image and the (d,e) height profiles corresponding to the lined regions in (c).

Next, we have conducted XPS to understand the chemical transformations during the APCVD process via the information on product's elements and their valence states (Figure 3). Thanks to the large X-ray beam size with a diameter of $400 \mu \mathrm{m}$, which can cover the whole dendrite, we are able to quantify the elemental ratio of $\mathrm{Pt}$ to $\mathrm{Mo}$ in the as-received hybrids by averaging 5 tested domains. Normalized atomic percentages can be acquired from the areas of the elemental peaks detected through a survey scan (Figure 3a), following background subtraction and application of sensitivity factors. The Pt content has been confirmed to be $5 \mathrm{wt} \%$ in this case. 
Apart from the characteristic peaks of Mo and S, the observation of Pt peaks validates its presence in the $\mathrm{MoS}_{2}$. The peak position values are displayed in Table S1, echoing the reported data. ${ }^{32,40,41}$ The $\mathrm{Mo}^{6+}$ ionization state is ascribed to the $\mathrm{MoO}_{3}$ precursor that has not been sulfurized during the APCVD or local oxidized regions of the sample after exposure to air. The Mo $3 \mathrm{~d}$ peaks of Pt:MoS $\mathrm{Mendrite}_{2}$ are downshifted by $0.3 \mathrm{eV}$ relative to those of pristine $\mathrm{MoS}_{2}$ (Figure 3b), indicating that the Mo atoms gain electrons as a consequence of the Pt incorporation. Moreover, downshifts of $0.2-0.3 \mathrm{eV}$ and upshifts of $0.2 \mathrm{eV}$ in the binding energy are respectively observed for the S 2p peaks (vs pristine $\mathrm{MoS}_{2}$, Figure 3c) and Pt 4f peaks (vs pure Pt metal, Figure S4e), which should result from the bonding effects between Pt and S. The modified electronic state density of $\mathrm{S}$ can improve the HER behavior of the neighboring Mo. ${ }^{42}$ The n-type doping in Mo is verified by the shifts of Mo 3d peaks (Figure 3b) ${ }^{43}$ which raises its hydrogen capturing ability. In contrast, first-principles density functional theory (DFT) calculations have indicated that Pt atoms would prefer binding to the Mo. This discrepancy may be attributed to the distinct hybridization routes. The computational study assumed that the Pt was deposited atop pre-synthesized $\operatorname{MoS}_{2},{ }^{30,44,45}$ whereas the experimental characterization was performed on the $\mathrm{Pt}$ doped simultaneously with the synthesis of $\mathrm{MoS}_{2} \cdot{ }^{43}$ As regards Pt nanoclusters, more favorably binding to the S network is unanimously agreed (Figure $3 c$ ), ${ }^{42,46}$ which may occur soon after they are formed because of their larger size and lower migration ability. ${ }^{7}$ Deriving from these electronic modifications, the valence band edge of $\mathrm{MoS}_{2}$ is lowered by $80 \mathrm{meV}$ from 0.74 to $0.66 \mathrm{eV}$ (Figure 3e). Similar effect has been observed in the manganese-doped $\mathrm{MoS}_{2}$, in which $\mathrm{Mn}-\mathrm{S}$ bond is formed. The substitutional magnetic element, Mn, accounts for a larger shift of $150 \mathrm{meV} .{ }^{37}$ The binding energy differences between the S $2 p$ doublet peaks and between the Pt $4 \mathrm{f}$ doublet peaks are changed by $0.1 \mathrm{eV}$ after the Pt decoration, which indicate that the formation of Pt-S bonds 
slightly affects the chemical states of these two elements (originally $\mathrm{S}^{2-}$ and $\mathrm{Pt}^{0}$ ). ${ }^{47}$ This also gives rise to the $\mathrm{Pt}^{\delta+}$ peaks deconvoluted via Lorentzian/Gaussian fitting, ${ }^{22}$ where $\delta \approx 2,{ }^{48}$ though the stronger $\mathrm{Pt}^{0}$ peak implies the dominance of $\mathrm{Pt}$ metal. The $\mathrm{Pt}^{\delta+}$ state is believed unrelated to the $\mathrm{Pt}$ salt residue, ${ }^{22}$ considering that the decomposition temperature of $\mathrm{H}_{2} \mathrm{PtCl}_{6}$ is far below those in our APCVD process. The binding in Pt:Mo alloy crystals is also analyzed by XPS (Figure S4 and Table S1), where the intermetallic bond induces positive core level shifts for both Pt and Mo. ${ }^{49}$

Our XPS results in Figure 3 and S4 suggest the preferred reaction pathways during the APCVD under different conditions, in accordance with the chemical thermodynamics and kinetics. The binding of Pt has been found to be dependent on its loading density. When there is abundant Pt on the substrate, the vaporous Mo would prefer to bind to the Pt, forming a bimetallic alloy. This is favored over the synthesis of $\mathrm{MoS}_{2}$, because the local supply of Pt on the GC substrate exceeds the feedstock of S transported by the carrier gas, and the mean free path of Mo to meet Pt is much shorter than that to meet $\mathrm{S}$. The alloyed Pt:Mo crystal is highly resistant to sulfur, ${ }^{50}$ and the impeded sulfurization causes negligible yield of Pt:Mo:S or $\mathrm{MoS}_{2}$. When the existing Pt is deficient, the Mo would first react with $\mathrm{S}$ owing to the lower activation energy, producing $\mathrm{MoS}_{2}$. Then, the $\mathrm{S}$ in the resultant $\mathrm{MoS}_{2}$ can form bonds with the Pt. In the latter case, the APCVD growth of $\mathrm{MoS}_{2}$ and its hybridization with Pt are two successive but independent processes. We determine by experiments that the threshold of Pt content impacting the thermodynamically favorable reactions is $\sim 5 \mathrm{wt} \%$. 

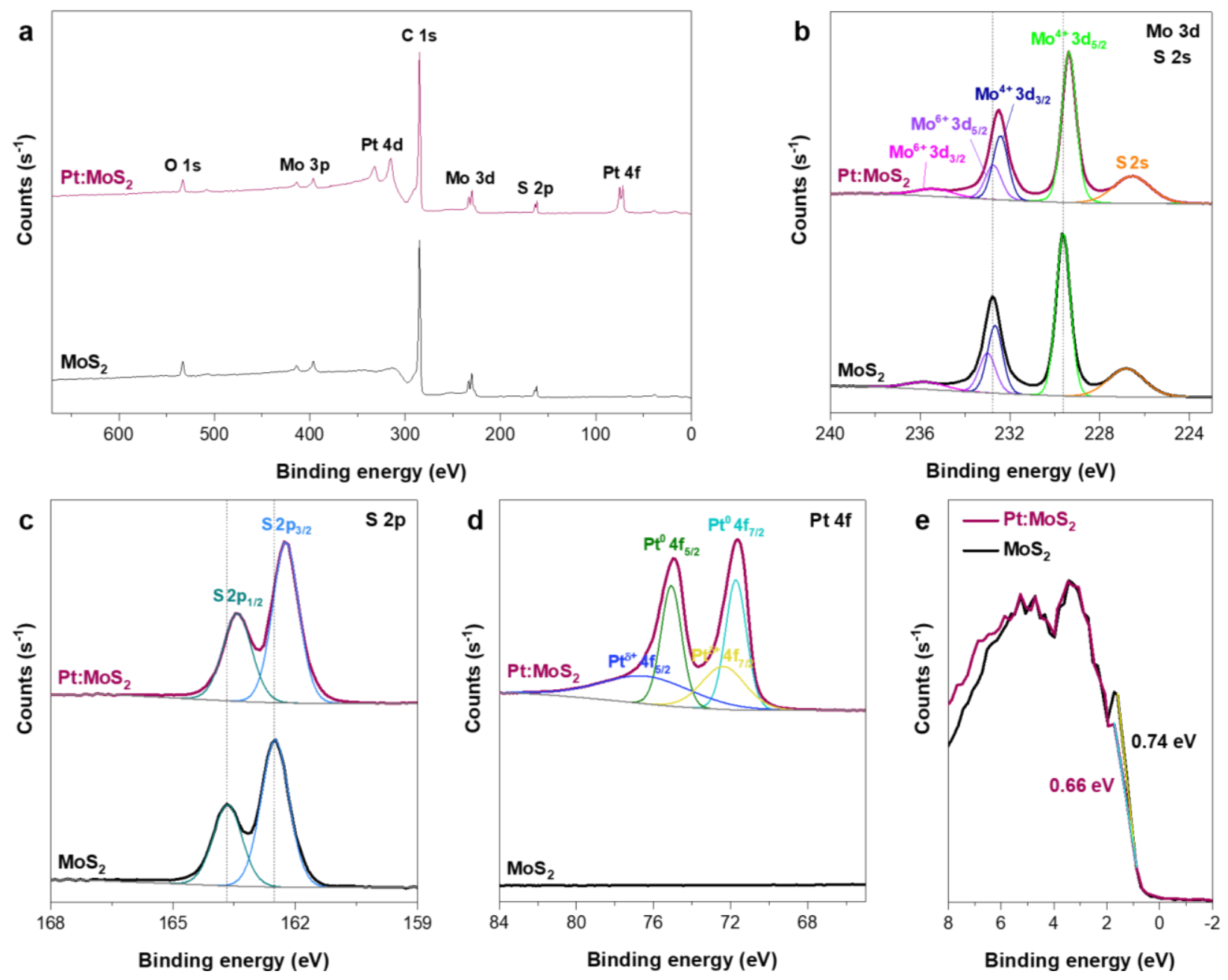

Figure 3. XPS spectra of 5 wt $\%$ Pt:MoS 2 vs pristine $\mathrm{MoS}_{2}$ monolayer dendrites on GC thin plates. (a) Survey scans across a wide range to show all the compositional elements. (b-d) Detailed scans for the elemental orbitals of (b) Mo 3d and S 2s, (c) S 2p, and (d) Pt 4f. The peaks are fitted after removing the background, with a combination of Lorentzian and Gaussian functions ( $\mathrm{L} / \mathrm{G}=30 \%)$ applied to obtain best fitting outcomes. (e) Valence-band spectra. Linear fits have been applied to show the valence band maximum (VBM) of $\mathrm{MoS}_{2}$ before and after Pt doping.

The Pt-decorated $\mathrm{MoS}_{2}$ has been imaged utilizing ADF-STEM to further investigate its atomic and nanoscale structure (Figure 4). At the loading amount of $1 \mathrm{wt} \%$, while a large number of the loaded Pt nanoclusters are dominant and highly dispersed across the $\mathrm{MoS}_{2}$ basal surface (Figure 
4a,b and Figure S6), single Pt atoms have also been achieved (Figure $4 \mathrm{f}$ and Figure S5b-d). An interlayer spacing of $0.24 \mathrm{~nm}$ has been measured from the corresponding fast Fourier transform (FFT) pattern, Figure 4c, which is the lattice spacing of a Pt nanocrystal in the (111) direction and consistent with the reported value. ${ }^{51}$ It demonstrates that Pt has been crystalized simultaneously with the growth of $\mathrm{MoS}_{2}$ dendrites. Besides, the reflections associated with the $\mathrm{MoS}_{2}$ and the Pt[111] align to each other, indicative of an epitaxy between these crystal systems. Consecutive scans reveal that the $\mathrm{Pt}$ atoms in Figure $4 \mathrm{~h}$ have migrated away from the region where they initially sat in Figure 4g. Therefore, they are surface-bound and not substituted in the lattice, that is, Pt does not replace Mo. The energy barrier for a Pt atom to migrate across the surface of a defect-free $\mathrm{MoS}_{2}$ monolayer is very low $(\sim 0.6-0.82 \mathrm{eV})$ at room temperature, so that random diffusion can be induce by the electron beam irradiation with a high accelerating voltage of $80 \mathrm{kV}$, till they are immobilized via bond formation. ${ }^{30,43-45}$ According to the theoretical prediction, the diffusion rate

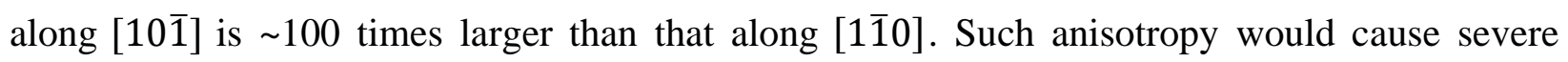
agglomeration of the Pt. ${ }^{44}$ Notwithstanding, monoatomic and homogeneous Pt distribution has been achieved by experiments (Figure $4 \mathrm{f}$ and $\mathrm{S} 5 \mathrm{~b}-\mathrm{d}$ ), in which the Pt doping proceeds together with the $\mathrm{MoS}_{2}$ synthesis. ${ }^{43}$ This highlights again the merit of our single-step growth strategy.

We have evaluated the diameter and specific surface area of the $\mathrm{MoS}_{2}$-supported $\mathrm{Pt}$ nanoparticles based on the ADF-STEM images of several different areas (Figure S6). The high magnification gives enough contrast from the single Pt atoms. By means of Gaussian fitting to the as-plotted distribution histogram in Figure 4i, the average size of the Pt particles is $\sim 5.7 \mathrm{~nm}$. A Gaussian function has been chosen here because it indicates normal distribution. As alternatives, the number averaged $\left(\bar{d}_{n}\right)$ and volume/area averaged $\left(\bar{d}_{\mathrm{v} / \mathrm{s}}\right)$ diameters have been calculated:

$$
\bar{d}_{n}=\frac{\sum d_{\mathrm{i}}}{\sum n_{\mathrm{i}}}=(5.2 \pm 0.9) \mathrm{nm}
$$




$$
\bar{d}_{\mathrm{v} / \mathrm{s}}=\frac{\sum n_{\mathrm{i}} d_{\mathrm{i}}{ }^{3}}{\sum n_{\mathrm{i}}{d_{\mathrm{i}}}^{2}}=5.8 \mathrm{~nm}
$$

The specific surface area (A) can be well estimated with a simplified equation:

$$
A=\frac{6}{\rho \bar{d}} \times 10^{3}=48.2 \mathrm{~m}^{2} \mathrm{~g}^{-1}
$$

where $\rho$ is the density of Pt metal, i.e., $21.45 \mathrm{~g} \mathrm{~cm}^{-2}, \bar{d}$ is the averaged particle diameter in $\mathrm{nm}$, and A usually takes the unit of $\mathrm{m}^{2} \mathrm{~g}^{-1}$. Note that $\bar{d}_{\mathrm{v} / \mathrm{s}}$ has been regarded preferable to $\bar{d}_{n}$ as a measure of $A .^{52}$ The chemical composition of our sample is reconfirmed by EDX (Figure $4 \mathrm{j}$ ). The peaks in the spectrum identify the existence of Pt dopants, Mo and $\mathrm{S}$ from $\mathrm{MoS}_{2}$, as well as $\mathrm{Si}$ from $\mathrm{Si}_{3} \mathrm{~N}_{4}$ holder. 


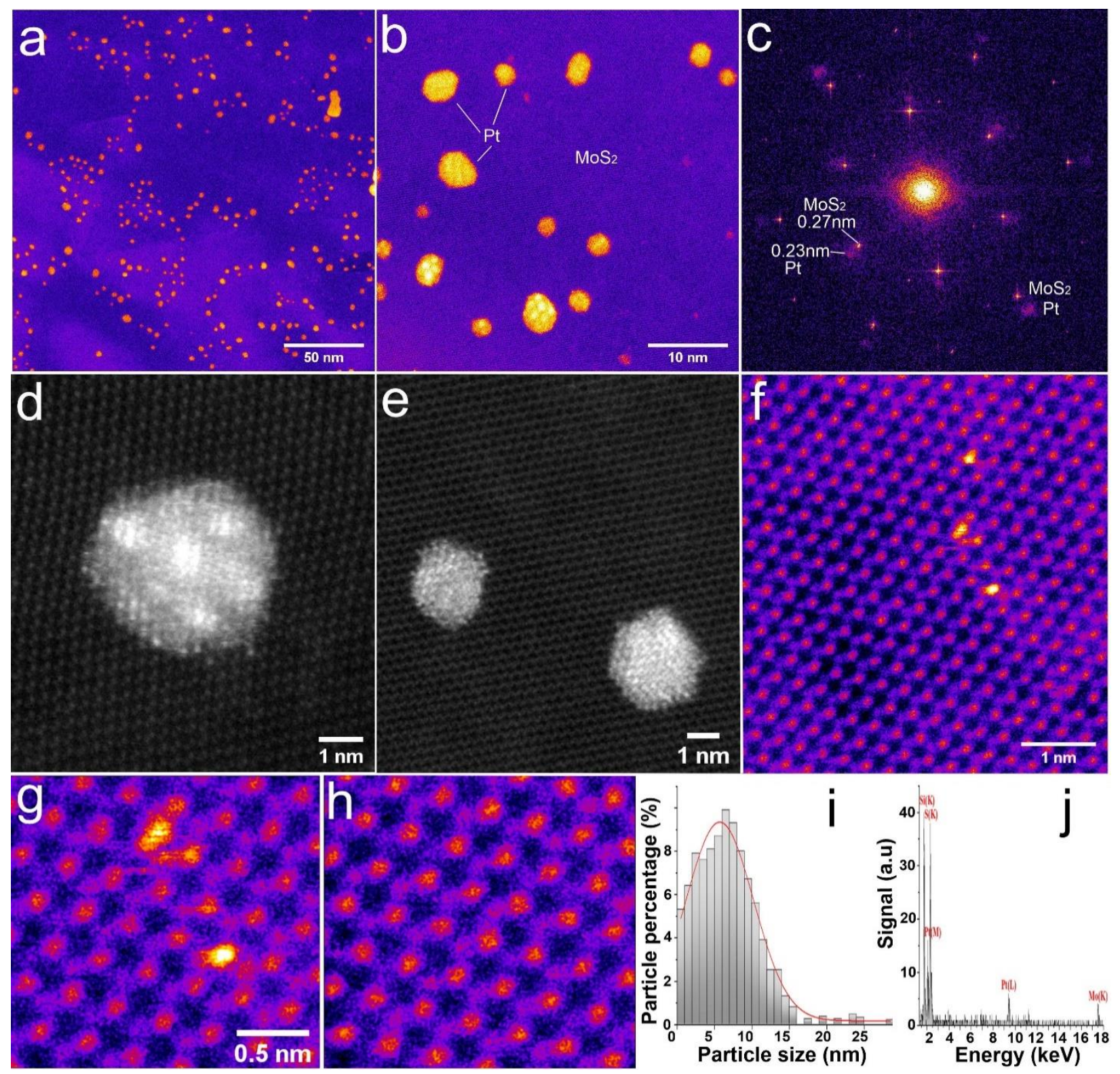

Figure 4. Structural and elemental characterization of the Pt:MoS 2 hybrid. (a,b) ADF-STEM images of the basal plane area under (a) lower and (b) higher magnifications. Pt nanoparticles are observed on top of the $\mathrm{MoS}_{2}$ surface. (c) FFT pattern from the entire image in (b). (d,e) ADF-STEM images of (d) one Pt nanocrystal and (e) two smaller Pt nanocrystals on monolayer $\mathrm{MoS}_{2}$. (f) High magnification image showing monoatomic Pt on the $\mathrm{MoS}_{2}$. (g,h) Images of the same region between consecutive scans. (i) EDX spectrum. The identified elements ( $\mathrm{Si}, \mathrm{S}, \mathrm{Pt}$, and $\mathrm{Mo})$ along with their respective electronic shells $(\mathrm{K}, \mathrm{M}$, and $\mathrm{L})$ are labelled above each peak. (j) Gaussian-fitted histogram illustrating the size distribution of the Pt particles. 
We have performed Raman and PL spectroscopies to further study the Pt-MoS 2 interactions and their effects. There are two Raman-active modes corresponding to $\mathrm{MoS}_{2}$ (Figure 5b), namely, $\mathrm{E}_{2 \mathrm{~g}}^{1}$ at $\sim 384.0 \mathrm{~cm}^{-1}$ for the in-plane $\mathrm{Mo}-\mathrm{S}$ phonon and $\mathrm{A}_{1 \mathrm{~g}}$ at $\sim 403.6 \mathrm{~cm}^{-1}$ for the out-of-plane $\mathrm{Mo}-\mathrm{S}$ phonon. The frequency difference of $A_{1 g}-E_{2 g}^{1}$ centering around $19 \mathrm{~cm}^{-1}$ lends further support to the monolayer thickness of the dendrite. ${ }^{53}$ Additionally, the Pt-caused blue shifts of both $\mathrm{A}_{1 \mathrm{~g}}$ and $\mathrm{E}_{2 \mathrm{~g}}^{1}$ peaks may arise from compressive strain and/or p-doping in the $\mathrm{MoS}_{2} .{ }^{54,55}$ The development of compressive strain is ruled out considering that the lattice constant of Pt is larger than that of in-plane $2 \mathrm{H}-\mathrm{MoS}_{2}$, and the p-doping will be reaffirmed based on the PL characteristics. The surface roughness of $\mathrm{MoS}_{2}$ can be suggested by the integrated Raman intensity ratio of $\mathrm{A}_{1 \mathrm{~g}} / \mathrm{E}_{2 \mathrm{~g}}^{1}$, as the $A_{1 g}$ and $E_{2 g}^{1}$ modes are preferentially excited for edge and terrace terminations, respectively. ${ }^{8}$ The higher ratios of the hybrids signify that the $\mathrm{Pt}$ decoration results in rougher surfaces.

Strong PL is observed in the monolayer $\mathrm{MoS}_{2}$ upon laser excitation owing to its direct bandgap, ${ }^{38}$ while comparatively, Pt doping leads to quenched peak intensity and red-shifted peak position (Figure 5c). To reveal the Pt influences on the exitonic emissions, we have applied Lorentzian fitting to the PL curves for increasing Pt contents (Figure 5d-f). Since localized states (LS) are induced by the structural disorders in $\mathrm{MoS}_{2}$ nanosheets, the enhancement of LS peak with increasing Pt content implicates the additional lattice defects introduced by the hybridization, which result in quenching and red shift of the PL peak. ${ }^{56}$ Another reason could be that the local electronic states are changed by the metallic Pt, thus affecting the band formation of $\mathrm{MoS}_{2} .{ }^{57}$ The presence of negatively charged $\mathrm{A}$ excitons ( $\mathrm{A}^{-}$, also known as trions) demonstrate the $\mathrm{n}$-doping in the $\mathrm{MoS}_{2}$, originating from the GC supporting substrate and/or the chemical binding to the Pt. The 
relative population densities of $\mathrm{A}$ and $\mathrm{A}^{-}$can be gained by comparing the integrated intensity of their peaks. ${ }^{58}$ The increasing $\mathrm{A} / \mathrm{A}^{-}$ratio with the $\mathrm{Pt}$ loading is suggestive of the Pt-induced $\mathrm{p}$ doping in the $\mathrm{MoS}_{2}$ under light excitation. This is because the Fermi level of Pt is lower compared with that of $\mathrm{MoS}_{2}$, so that a band offset is formed at the $\mathrm{Pt}-\mathrm{MoS}_{2}$ heterojunction and the electrons are transferred from the $\mathrm{MoS}_{2}$ semiconductor to the Pt metal upon photoinduction. ${ }^{22}$ The changes in the $\mathrm{MoS}_{2}$ spectroscopic features caused by the Pt decoration therefore offer feasibilities of roughly probing the Pt location via Raman and PL mapping. It is notable that the quenching at the $\mathrm{MoS}_{2}$ edges seen from the Raman and PL intensity maps has no association with the loaded Pt (Figure 5g,h), since this is a typical phenomenon for TMD isolated domains, relying on the smallest laser spot size that can be reached, i.e., $\sim 1 \mu \mathrm{m}$ by our equipment. ${ }^{59}$ The grain boundaries on the dendrite basal surface cannot be visualized in this way because of our detection limit, otherwise we might observe increased PL intensity and decreased Raman intensity at the same position. ${ }^{60}$ Nevertheless, this does not affect the identification of Pt nanoclusters sitting on the grain boundaries. The differentiated A-exciton PL peak intensity and position (Figure 5h,i), as well as the Raman peak intensity, which is less affected due to the relatively weak signals (Figure $5 \mathrm{~g}$ ), manifest that Pt has decorated the basal plane of the $\mathrm{MoS}_{2}$ dendrite, consistent with the ADFSTEM observations (Figure 4, Figure S5 and Figure S6). In comparison, pristine $\mathrm{MoS}_{2}$ dendrite shows uniform Raman and PL signals across its surface. ${ }^{10}$ 

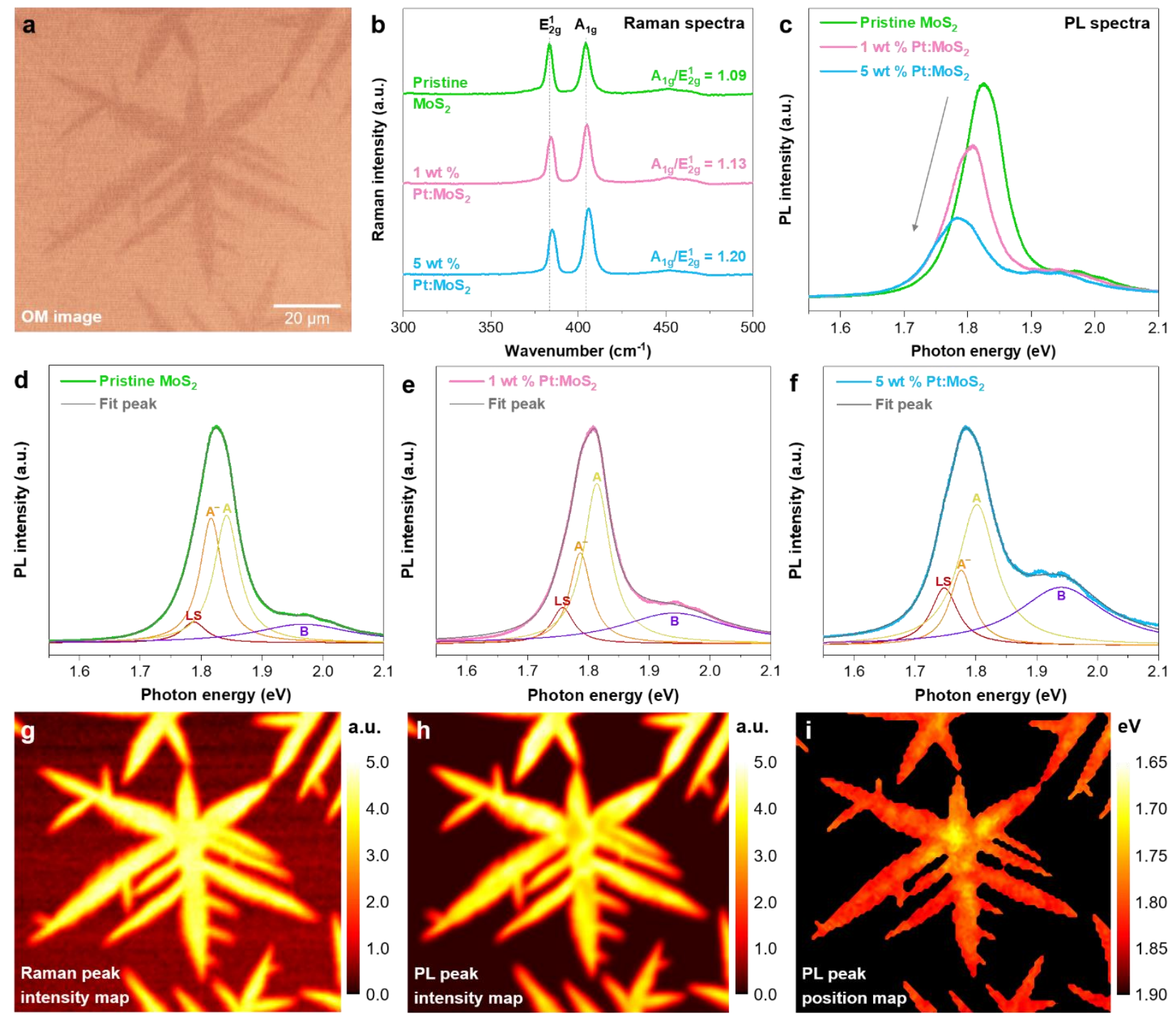

Figure 5. Raman and PL spectroscopies of the CVD-grown dendritic monolayer Pt:MoS $\mathrm{S}_{2}$ hybrids and pristine $\mathrm{MoS}_{2}$. (a) Optical microscopic image of a typical dendrite for the measurements. (b) Raman and (c) PL spectra. (d-f) Deconvolution for the PL curves in (c), which correspond to (d) pristine $\mathrm{MoS}_{2}$, (e) 1 wt \% Pt:MoS 2 , and (f) 5 wt \% Pt:MoS 2 , revealing the emissions of B excitons (B), A excitons (A), negatively charged A excitons $\left(\mathrm{A}^{-}\right)$, and localized states (LS). Lorentzian functions are utilized for best fitting results, with each coefficient of determination $R^{2} \geq 0.9999$. (g-i) $2 \mathrm{D}$ maps of the dendrite in (a), showing (g) $A_{1 g}$ Raman peak intensity, (h) A-exciton PL peak intensity, and (i) A-exciton PL peak position. 
The electrocatalytic performances of the hybridized $\mathrm{Pt}: \mathrm{MoS}_{2}$ and pristine $\mathrm{MoS}_{2}$ grown on GC plates with dendritic and triangular shapes have been examined for the HER (Figure 6). All of these $\mathrm{MoS}_{2}$ are monolayer, ensuring that the interlayer charge barrier is minimal and consistent. Here, we do systematic analysis and provide new insights from several aspects.

Individual Catalytic Roles of Pt and $\mathrm{MoS}_{2}$ and Synergistic Effects in the Hybrid. As shown in Figure 6a,b, the enhanced catalysis by loading Pt (Pt:MoS $\left.\mathrm{Ss}_{2} \mathrm{MoS}_{2}\right)$ and by using dendritic scaffold (Pt:MoS 2 dendrites vs Pt:MoS 2 triangles) suggests that Pt works in tandem with $\mathrm{MoS}_{2}$ for the HER. The superior activities of the hybrids can be explained by two synergies in this system. One occurs between the atomically dispersed Pt species and the Pt(111) nanoclusters. Pt single atoms display maximized specific surface area, whereas Pt nanoclusters show higher conductivity and more efficient HER pathway (Heyrovsky route for Pt atoms vs Tafel route for Pt nanoclusters), hence their copresence on $\mathrm{MoS}_{2}$ with small separation distance could lead to increased catalytic activities. ${ }^{61}$ The other one is between $\mathrm{Pt}$ and $\mathrm{MoS}_{2},{ }^{7,42,62,63}$ manifested by the rapid hydrogen generation stages of $1 \mathrm{wt} \%$ and $5 \mathrm{wt} \% \mathrm{Pt}: \mathrm{MoS}_{2}$ dendrites (after reaching the overpotentials marked by the navy and olive dashed lines in Figure 6a). For the $\mathrm{MoS}_{2}$, the binding of $\mathrm{Pt}$ to $\mathrm{S}$ would open up its nearest Mo on the basal plane as a new electrocatalytic center. ${ }^{5,64,65}$ Furthermore, this bonding behavior could enhance the catalytic properties of both the edge-Mo and the surface-Mo by donating electrons to them (Figure 3b). ${ }^{42}$ For the Pt, the $\mathrm{MoS}_{2}$ offers stabilization sites. When the loading density is low, the Pt would preferentially nucleate and grow or aggregate at the unsaturated sites of $\mathrm{MoS}_{2}$, including its edges and grain boundaries (Figure $2 \mathrm{~b}, \mathrm{c}){ }^{7,33}$ At high Pt content, even dispersion is achieved across the $\mathrm{MoS}_{2}$ basal surfaces and edges (Figure $6 \mathrm{~g}) .{ }^{7}$ However, computational analysis has suggested that the metal-support interactions would not affect the HER mechanism or the catalytic activity in a $\mathrm{Pt}_{20}: \mathrm{MoS}_{2}$ system. ${ }^{66}$ The reason 
for this disagreement could be that the $\mathrm{Pt}_{20}$ is $\sim 6$ times on average smaller than the Pt nanoclusters dominantly existing in our hybrids. Hence, the $\mathrm{Pt}_{20}-\mathrm{Pt}_{20}$ distance is much shorter for a certain loading amount of $\mathrm{Pt}$, leading to promoted Tafel reaction. ${ }^{61}$ Figure S8 summarizes the contributions of $\mathrm{Pt}$ and $\mathrm{MoS}_{2}$ alone and their synergies to the overall HER catalysis.

HER Kinetics and Mechanism. (1) Onset potential $(\eta)$ is an indicator of the catalyst's activation energy for the HER and can reflect the modulated kinetics. It can be obtained from the Tafel plot in Figure 6b, by identifying the point where the linear relationship $\eta=b \log |j|+a$ begins. Now we focus on the impacts of Pt loading on the behavior of either triangle or dendrite catalysts. The decreasing $\eta$ with the Pt content is a sign of the change in electrocatalytically active sites (Figure 6a). The LSV curves indicate that as the overpotential increases, the hydrogen evolution from 1 wt $\%$ Pt:MoS 2 can be divided into three stages, whereas two stages are shown by 0.1 wt $\%$ and $5 \mathrm{wt} \% \mathrm{Pt}: \mathrm{MoS}_{2}$. For all hybrids, the end of the first stage corresponds to the start of hydrogen production (olive dashed line), and the obviously reduced $\eta$ signifies the crucial role of Pt in the electrocatalysis. The second stage of $0.1 \mathrm{wt} \%$ and $1 \mathrm{wt} \% \mathrm{Pt}: \mathrm{MoS}_{2}$ is basically the result of the sole contributions from Pt and $\mathrm{MoS}_{2}$ entities. The third stage of $1 \mathrm{wt} \% \mathrm{Pt}: \mathrm{MoS}_{2}$ (navy dashed line) and the second stage of $5 \mathrm{wt} \% \mathrm{Pt}: \mathrm{MoS}_{2}$ exhibit fast release of hydrogen, which can be attributed to the synergistic effects arisen between $\mathrm{Pt}$ and $\mathrm{MoS}_{2}$ in addition to their own performances. These imply that a certain overpotential is required to activate the synergies, and its value reduces with increase in the Pt content. Despite the larger Pt amount, $5 \mathrm{wt} \% \mathrm{Pt}: \mathrm{MoS}_{2}$ seems to have comparable synergistic effects with those in $1 \mathrm{wt} \% \mathrm{Pt}: \mathrm{MoS}_{2}$, leading to similar slopes of their LSV curves at high overpotentials. This can be interpreted by the decoration of Pt nanoclusters on the $\mathrm{MoS}_{2}$ edges and defects. Otherwise such catalytic active regions along with the derived synergistic effects would be retained by their exposure. It is noteworthy that for $0.1 \mathrm{wt} \% \mathrm{Pt}: \mathrm{MoS}_{2}$, some synergistic 
behaviors might also take place at increased overpotential, but with a small amount Pt interacting with the $\mathrm{MoS}_{2}$, noticeable improvements in the HER efficiency are unlikely to be brought. (2) Tafel slope can suggest the hydrogen evolution rate. It has specific values referring to different elementary reactions: $120 \mathrm{mV} \mathrm{dec}^{-1}$ for Volmer (adsorption of $\mathrm{H}_{3} \mathrm{O}^{+}$onto a catalytic active site: $\mathrm{H}_{3} \mathrm{O}^{+}+\mathrm{e}^{-} \rightarrow \mathrm{H}^{*}+\mathrm{H}_{2} \mathrm{O}$ ), $40 \mathrm{mV} \mathrm{dec}{ }^{-1}$ for Heyrovsky (hydrogen desorption from the catalyst site through an electrochemical reaction: $\mathrm{H}^{*}+\mathrm{H}_{3} \mathrm{O}^{+}+\mathrm{e}^{-} \rightarrow \mathrm{H}_{2}+\mathrm{H}_{2} \mathrm{O}$ ), and $30 \mathrm{mV} \mathrm{dec}{ }^{-1}$ for Tafel (desorption of $\mathrm{H}_{2}$ from the catalytic site via combination of two adsorbed hydrogen intermediates: $\mathrm{H}^{*}+\mathrm{H}^{*} \rightarrow \mathrm{H}_{2}$ ) routes of water-splitting. Volmer-Tafel and Volmer-Heyrovsky are the recognized HER pathways in acidic media. The Tafel slope is evidently decreased after incorporating Pt (Figure 6b), e.g., from 112 to $46 \mathrm{mV} \mathrm{dec}^{-1}$ by loading 5 wt $\%$ Pt onto the $\mathrm{MoS}_{2}$ dendrites, which indicates a combination of Volmer-Heyrovsky steps for such hybrid cocatalysts. The Tafel plots of 0.1 and 1 wt \% Pt:MoS 2 show "curvy" portions with linear Tafel-slope portions in the middle, while these should not be linked to the transition of hydrogen evolution stages. To prove it, we show the results over a wide range (Figure S7), where no more linear correlation is observed. That is, in each plot there is only one approximation to the Butler-Volmer equation and therefore each catalyst has only one Tafel slope. (3) Exchange current density $j_{0}$ is commonly used to describe the efficiency of electron transfer in catalysts at zero overpotential. So, it should increase with the improved hybrid-substrate contact resulting from the direct growth and thermal annealing ${ }^{19}$ which will be ascertained later. The value is calculated by fitting the linear portion of the Tafel plot $(\log j$ vs $\eta) .5,67$ 

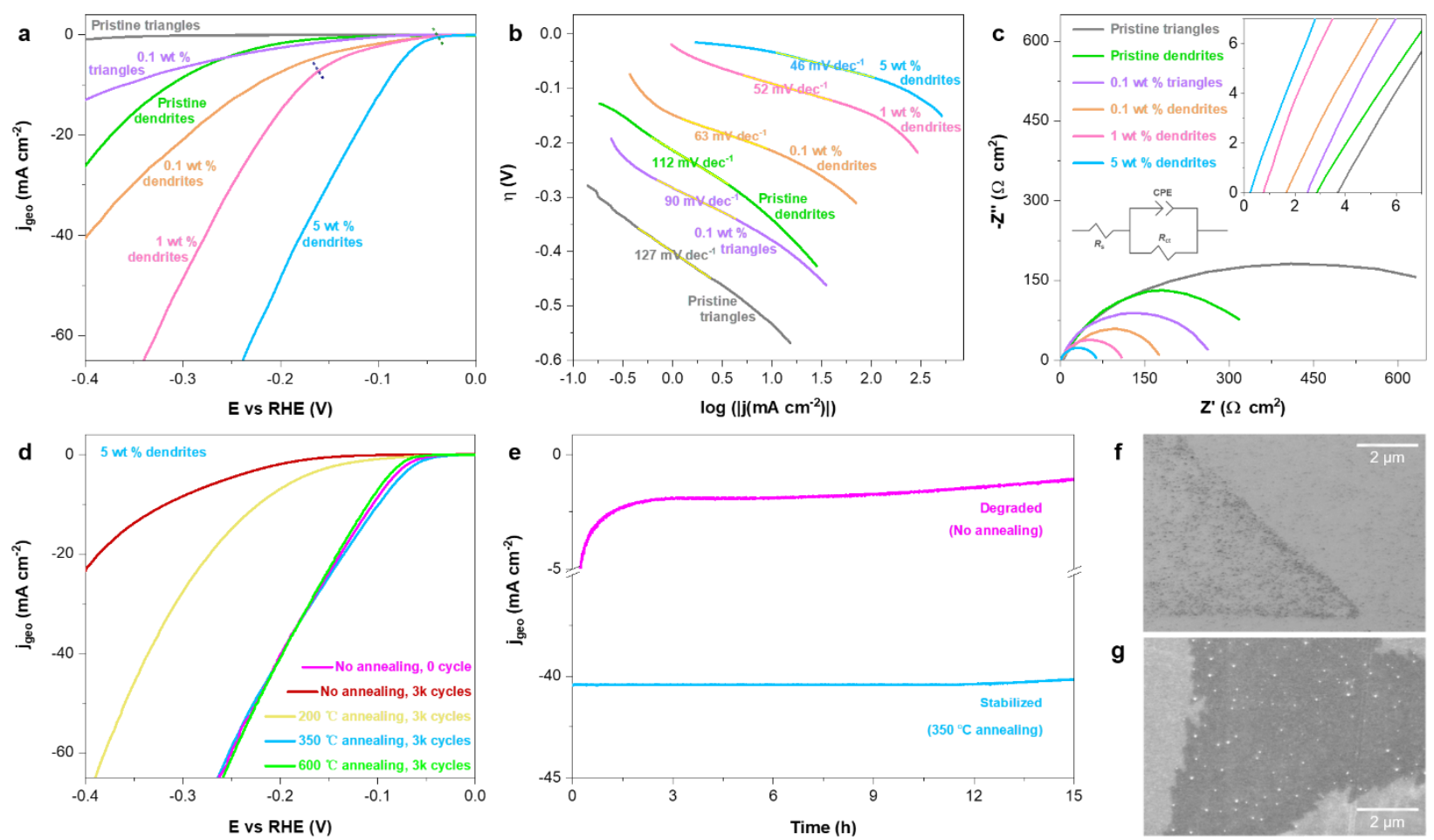

Figure 6. HER activities of the dendritic and triangular monolayer $\mathrm{MoS}_{2}$ with varying Pt contents. (a) Cathodic polarization curves. The current density is normalized to the geometric area of the GC working electrode, denoted by the subscript of "geo". The transitions in hydrogen evolution stages are marked by the olive and navy dashed lines. (b) Tafel plots with the slopes obtained via linear fits. (c) Nyquist plots at $10 \mathrm{mV}$ DC bias with the frequency swept from 200000 to $1 \mathrm{~Hz}$, which shows the impedance of the electrochemical system. The left lower inset is a Randles equivalent circuit model, and the right upper inset shows the zoomed-in high frequency region (squared). (d-g) Stability investigation on the $5 \mathrm{wt} \%$ dendrite hybrids. (d) Cathodic polarization curves before and after 3,000 cycles for different annealing treatments. (e) Chronoamperometric responses at the overpotential of $-0.2 \mathrm{~V}$ vs RHE and (f,g) SEM images, which compare the stabilized with the unstabilized samples as a result of long-term cycling.

We correlate these electrocatalytic metrics of the dendrite hybrids to the $\mathrm{Pt}$ content quantified by wt $\%$ and $\mu \mathrm{g} \mathrm{cm}^{-2}$ (Figure $7 \mathrm{a}-\mathrm{c}$ ). The numbers are listed in Table 1 . The dependence of Pt surface area on its loading percentage is substantiated by investigating the current density of 
the dendrite hybrids at three overpotentials, i.e., $-0.1,-0.2$, and $-0.3 \mathrm{~V}$ vs RHE (Figure $7 \mathrm{~d}-\mathrm{f}$ ). When more $\mathrm{Pt}$ is loaded, on one hand, the Pt species would agglomerate into relatively larger particles,${ }^{44}$ so that both its activity per atom and the synergy between Pt nanoclusters and Pt single atoms are diminished. It is worth mentioning that the Pt migration could be facilitated as the overpotential decreases owing to the propulsion by the increasing amount of the generated $\mathrm{H}_{2}$ bubbles. ${ }^{68}$ Yet, on the other hand, the synergy between Pt and $\mathrm{MoS}_{2}$ should be enhanced. This is because the total number of the active sites increases with the Pt loading, signified by the decreasing onset potential as a function of the Pt content (Figure 7a) and the increasing HER performance (Figure 6a,b). At low overpotential of $-0.1 \mathrm{~V}$ vs RHE, the Pt-MoS synergy is only activated with the highest Pt loading, verified by the increased hydrogen evolution rate beyond the critical overpotential (olive dashed line in Figure 6a). The synergistic behavior in the 5 wt $\%$ Pt: $\mathrm{MoS}_{2}$ dendrites is dominant over the agglomeration of $\mathrm{Pt}$, and therefore the current density exhibits a superlinear trend with the Pt loading (Figure 7d). Since at high overpotential of $-0.3 \mathrm{~V}$ vs RHE, the synergies are switched on for both 1 wt $\%$ and 5 wt $\%$ Pt loadings (navy and olive dashed lines in Figure 6a, respectively), the impacts of the Pt size would surpass that of the Pt$\mathrm{MoS}_{2}$ synergy, leading to a sublinear relationship (Figure 7f). These competing mechanisms turn out to deliver an apparent linear curve at $-0.2 \mathrm{~V}$ vs RHE (Figure 7e). 

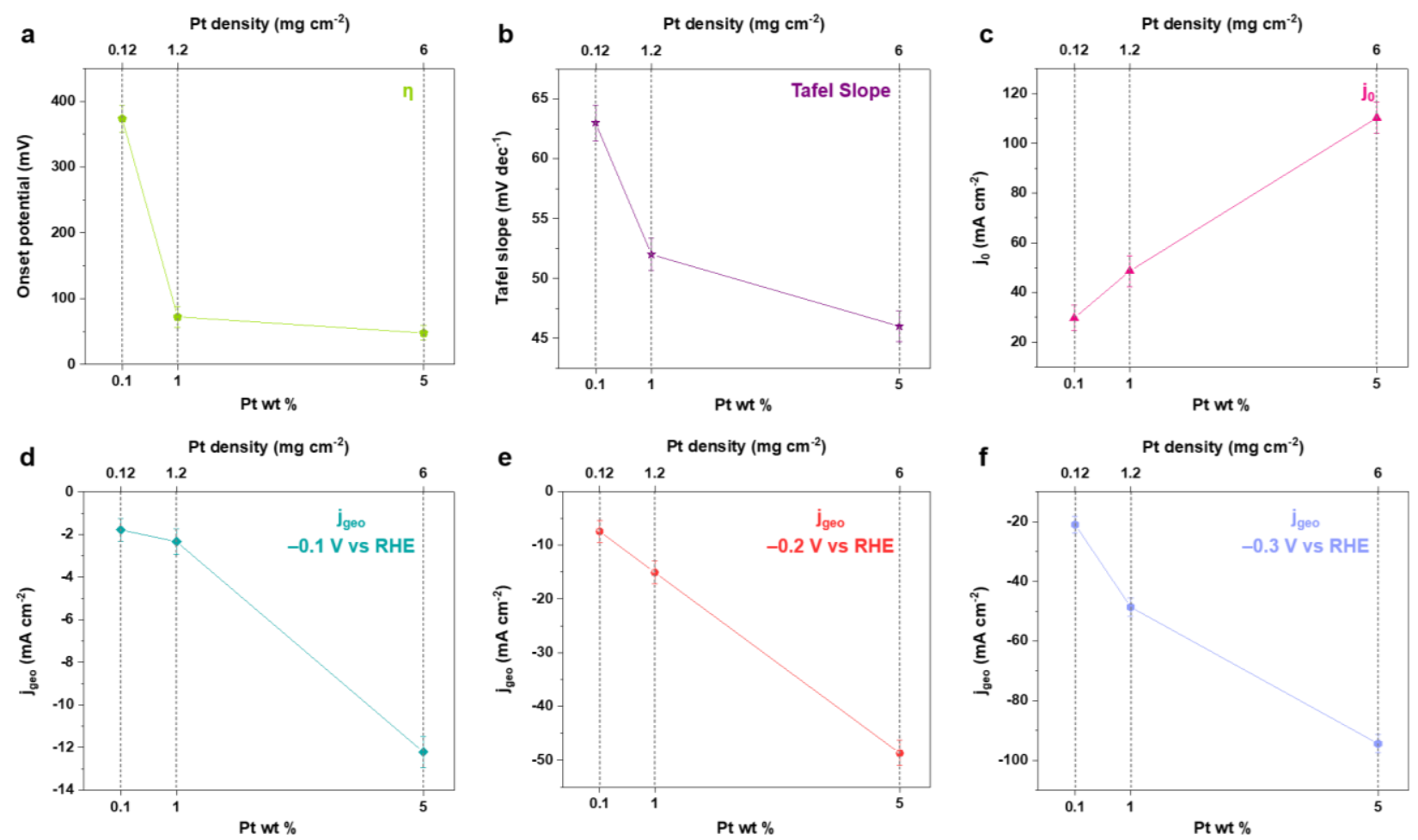

Figure 7. Pt content dependence of the HER activities in Pt:MoS ${ }_{2}$ dendrite hybrids, with regards to the (a) $\eta$, (b) Tafel slope, (c) $j_{0}$, current density at (d) $-0.1 \mathrm{~V}$, (e) $-0.2 \mathrm{~V}$, and (f) $-0.3 \mathrm{~V}$ vs RHE. The deviations from averaged values could stem from the areal coverage fluctuations of the dendrites.

Conductivity. The electrical conductivity of the catalyst system is measured by electrochemical impedance spectroscopy (EIS), Figure 6c. Within the frequency range of 200000 to $1 \mathrm{~Hz}$, the semiarc at low frequency region indicates the kinetic control in the electrode process. Mass transport control is excluded, otherwise a $45^{\circ}$ straight line would be shown. The absence of another semiarc at high frequency region is due to the decreased electron tunneling barrier between the directly grown catalyst layer and the GC electrode compared to that for the $\mathrm{MoS}_{2}$ transferred on it. ${ }^{10,19,69}$ The better interfacial contact accounting for this benefits from the direct growth on the electrode and the thermal annealing treatment. Also, the $x$-axis intercept represents the ohmic impedance of the electrochemical reaction system. ${ }^{70}$ The smaller semiarc radius and smaller $x$ - 
intercept reveal the lower charge transfer resistance $\left(R_{\mathrm{ct}}\right)$ at the hybrid-electrolyte interface and the lower series resistance $\left(R_{\mathrm{S}}\right)$ of the cocatalyst, respectively, which is attributable to both the metallicity of Pt and the dendritic morphology (containing more electron hopping sites) of $\mathrm{MoS}_{2}$.

Durability. To simulate the realistic operation of water-splitting devices, our dendritic monolayer Pt:MoS 2 cocatalysts have been subjected to durability tests through LSV at a fast scan rate of $100 \mathrm{mV} \mathrm{s}^{-1}$ in the same aqueous environment $\left(0.5 \mathrm{M} \mathrm{H}_{2} \mathrm{SO}_{4}\right)$ for the HER. The Pt:MoS hybrid untreated with annealing exhibits low stability upon cycling (Figure 6d). The degradation is likely to stem from the behavior of Pt nanoclusters as follows: ${ }^{68}$ (1) Ostwald ripening, by which small metal crystals dissolve and then redeposit onto larger crystals. (2) Aggregation, occurring when the Pt particles migrate across the surfaces of $\mathrm{MoS}_{2}$ as well as the GC plate via Brownian motion. Both (1) and (2) would cause Pt sintering with a reduction in the active surface area, and thereby hampering its electrocatalysis and stability. (3) Detachment from the GC electrode, as the Pt nanoclusters could be prone to subtle disturbance in fluids. To mechanistically understand the degradation, we have probed the chemical bonding $\left(\mathrm{Pt}-\mathrm{MoS}_{2}\right)$ and physical adhesion $\left(\mathrm{Pt}: \mathrm{MoS}_{2}\right.$ hybrids to GC electrode) by XPS (Figure S9) and SEM imaging (Figure 6f,g), in conjunction with the electrochemical measurements (Figure 6d,e). For the non-annealed sample, the SEM image indicates the cycling-induced loss of both $\mathrm{Pt}$ and $\mathrm{MoS}_{2}$ (Figure 6f). This is supported by the increase in $\eta$ (Figure 6d), reflects the altered catalytic sites, as well as the intensity quenching of Mo 3d, S 2p, and Pt 4f peaks in the XPS spectra (Figure S9). The Mo 3d and S 2p peaks shift farther away from those of pristine $\mathrm{MoS}_{2}$, and the $\mathrm{Pt} 4 \mathrm{f}$ peaks shift towards $\mathrm{Pt}^{\delta+}$, signifying that a higher percentage of the preserved Pt is chemically bound to the $\mathrm{MoS}_{2}$, and the detached Pt is more likely to have been physically adsorbed on the $\mathrm{MoS}_{2}$. The increased percentage of the S-bound Pt after cycling is also verified by the larger position differences between the $\mathrm{Pt} 4 \mathrm{f}$ peaks along with 
the smaller position differences between the S 2p peaks (Table S2). The Mo $3 \mathrm{~d}$ peaks are redshifted due to the n-doping induced by the binding of its nearest neighboring $\mathrm{S}$ to $\mathrm{Pt}$, while the position difference between them stays unchanged, implicating again the absence of Pt-Mo bonds in our system. Notably, if Ostwald ripening and aggregation of the Pt occur before detachment, the $\mathrm{Pt} 4 \mathrm{f}$ peaks might shift intermediately towards $\mathrm{Pt}^{0}$ because of the weakened chemisorption to the $\mathrm{MoS}_{2}$. But these are not the eventually dominant causes for the deteriorated HER performance over the materials loss.

Aiming to enhance physisorption of the hybrid to the electrode surface and obtain durable catalysts for the HER, we have involved a thermal annealing stage in the APCVD process (Figure 1c). The HER activities of the 5 wt $\% \mathrm{Pt}: \mathrm{MoS}_{2}$ dendrite hybrid annealed under the optimized conditions is stable after 3,000 cycles $\left(350-600{ }^{\circ} \mathrm{C}\right.$ for $\left.25 \mathrm{~min}\right)$. Increasing the temperature can enhance the physical adsorption between Pt and its $\mathrm{MoS}_{2}$ scaffold, ${ }^{71}$ whereas this would reduce the free energies of Ostwald ripening and aggregation. Therefore, the aforementioned three processes should compete against one another at an elevated temperature, assuming that more than one would happen. The stronger physisorption at the $\mathrm{Pt}-\mathrm{MoS}_{2}$ interface plays a predominant role within this temperature range over the facilitated Ostwald ripening and aggregation of $\mathrm{Pt}$ nanocrystals, aside from the enhanced chemisorption of Mo-edges to the GC support, which might exist and could lead to weaker hydrogen binding. ${ }^{72}$ Lower temperature cannot guarantee the sufficient adhesion enhancement (Figure 6d), and further increasing the temperature above $600{ }^{\circ} \mathrm{C}$ would cause the decomposition of $\mathrm{MoS}_{2} \cdot{ }^{41}$ The improved long-term stability $(\sim 12 \mathrm{~h})$ is validated by chronoamperometry (Figure 6e) and SEM imaging (Figure 6g). ${ }^{42}$ We have not seen changes in the XPS spectra before and after annealing, which means that the chemisorption between Pt and 
$\mathrm{MoS}_{2}$ is unaffected during this treatment. The reason is that the annealing temperature is much lower than that of the main growth where most chemical bonds are formed.

Outperformance of Dendrite. The dendritic $\mathrm{MoS}_{2}$ scaffold is advantageous over the triangular counterpart in three aspects. (1) Higher densities of catalytic centers: the dendrite surface is enriched with $\mathrm{S}$ defects and undercoordinated Mo atoms. Both of these sites exhibit HER activities. ${ }^{5,14,25,64,65}$ (2) Preferences of Pt seeding: at reduced Pt density, the edges and defects are the energetically more favorable sites compared with the lattice plane to stabilize the $\mathrm{Pt}^{7,30,32,33}$ Besides, the dendrites possess a $23 \%$ larger surface area than the triangles, as indicated by the SEM images in Figure $2 \mathrm{~b}$ and Figure S2. This would allow more Pt atoms to be bound on the $\mathrm{MoS}_{2}$ and enable more interactions between them. (3) Increased conductivity: the higher densities of exposed edges and defects can bring more pairing chances of the hopping $\mathrm{e}^{-}$on the electrode and the $\mathrm{H}_{3} \mathrm{O}^{+}$in the electrolyte to form chemisorbed $\mathrm{H}^{*}$, and the promoted Pt stabilization can reduce the ohmic impedance of the reaction system, which are evidenced by its lower $R_{\mathrm{ct}}$ and $R_{\mathrm{s}}$ (Figure 6c), respectively. Based on these, the advantages of dendrite are embodied in both the hydrogen evolution catalysis of $\mathrm{Pt}$ and $\mathrm{MoS}_{2}$ themselves and the synergies in this cocatalyst system (Figure S8). The enhanced synergies in our dendritic-shaped hybrids can be confirmed by assessing the kinetic parameters for the HER: (1) the value differences between $0.1 \mathrm{wt} \% \mathrm{Pt}: \mathrm{MoS}_{2}$ dendrites and pristine $\mathrm{MoS}_{2}$ dendrites are larger than those between $0.1 \mathrm{wt} \% \mathrm{Pt}: \mathrm{MoS}_{2}$ triangles and pristine $\mathrm{MoS}_{2}$ triangles; (2) the value differences between $0.1 \mathrm{wt} \% \mathrm{Pt}: \mathrm{MoS}_{2}$ dendrites and 0.1 wt $\%$ Pt:MoS 2 triangles are larger than those between pristine $\mathrm{MoS}_{2}$ dendrites and pristine $\mathrm{MoS}_{2}$ triangles; (3) the $j_{0}$ of $0.1 \mathrm{wt} \% \mathrm{Pt}: \mathrm{MoS}_{2}$ dendrites is higher than the sum of that of pristine $\mathrm{MoS}_{2}$ dendrites and $0.1 \mathrm{wt} \% \mathrm{Pt}: \mathrm{MoS}_{2}$ triangles. The increment in $j_{0}$ reveals that the electron transfer is accelerated by employing $\mathrm{MoS}_{2}$ dendrites to accommodate the Pt. As a consequence, the rates of 
the Volmer and Heyrovsky reactions are both increased, leading to an enhancement in the HER efficiency.

Table 1. Key parameters for HER of the pristine and Pt-loaded $\mathrm{MoS}_{2}$ monolayer domains in this study. The averages are taken from 4 samples

\begin{tabular}{lccc}
\hline & $\begin{array}{c}\text { Onset potential } \eta \\
(\mathrm{mV})\end{array}$ & $\begin{array}{c}\text { Tafel slope } \\
\left(\mathrm{mV} \mathrm{dec}^{-1}\right)\end{array}$ & $\begin{array}{c}\text { Exchange current density } j_{0} \\
\left(\mu \mathrm{A} \mathrm{cm}^{-2}\right)\end{array}$ \\
\hline Pristine $\mathrm{MoS}_{2}$ triangles & $525 \pm 34.8$ & $127 \pm 3.9$ & $0.7 \pm 0.1$ \\
Pristine $\mathrm{MoS}_{2}$ dendrites & $487 \pm 29.6$ & $112 \pm 3.5$ & $18.5 \pm 2.0$ \\
$0.1 \mathrm{wt} \% \mathrm{Pt}: \mathrm{MoS}_{2}$ triangles & $419 \pm 27.1$ & $90 \pm 3.0$ & $4.2 \pm 0.3$ \\
$0.1 \mathrm{wt} \% \mathrm{Pt}: \mathrm{MoS}_{2}$ dendrites & $374 \pm 20.7$ & $63 \pm 1.5$ & $29.7 \pm 5.1$ \\
$1 \mathrm{wt} \% \mathrm{Pt}: \mathrm{MoS}_{2}$ dendrites & $72 \pm 16.3$ & $52 \pm 1.4$ & $48.6 \pm 6.2$ \\
5 wt \% Pt: $\mathrm{MoS}_{2}$ dendrites & $48 \pm 11.2$ & $46 \pm 1.3$ & $110.3 \pm 6.4$ \\
\hline
\end{tabular}

Comparisons with Literature. The key HER electrocatalytic parameters of the lowdimensional Pt:MoS 2 reported to date are summarized in Table S3. There are variations in the materials interactions, morphologies, defect and strain states, synthetic approaches, etc. We have been dedicated to developing a single-step approach to produce directly applicable catalysts, improving the HER performance, increasing the stability, and discovering the catalytic mechanism including the synergistic effects in our unique system. Comparative analysis regarding the HER performance outlines five reasons for the high density of catalytic sites on the dendrite hybrids in the present study vs the previously reported Pt:MoS 2 with similar doping percentages. ${ }^{43}$ (1) The monolayer thickness of the $\mathrm{MoS}_{2}$ that we have grown leads to decreased potential barrier for electron hopping within the interlayer gap and hence increased efficiency of charge transfer from the electrode,${ }^{35}$ consequently facilitating the hydrogen evolution process. (2) The dendritic scaffold 
contains plenty of edges and grain boundaries, both of which intrinsically exhibit electrocatalysis

for the HER. (3) These featured sites could show higher adsorption energy for the Pt species, ${ }^{7,33}$ so that the Pt is more easily seeded and stabilized on the dendritic $\mathrm{MoS}_{2}$. (4) Enhanced synergistic effects between Pt and $\mathrm{MoS}_{2}$ are achieved owing to the improvement in Pt density and bonding strength. ${ }^{7,42,62,63}$ (5) Synergy also emerges among the highly dispersed Pt species. This is attributed to the short separation distances between small Pt nanoclusters and single Pt atoms, which result in increased electron channels (hence conductivity) and Pt specific surface area (hence utilization ratio). ${ }^{61}$ Overall, the improved HER activities of our dendrite hybrids mainly benefit from the morphology design of the $\mathrm{MoS}_{2}$ and Pt.

\section{CONCLUSION}

In summary, Pt:MoS 2 cocatalyst systems have been constructed utilizing single-step APCVD approach. We have determined the loading threshold for producing Pt:MoS 2 hybrids against Pt:Mo alloys. The growth mode has been controlled to be either thermodynamic or kinetic, yielding either triangular or dendritic structures. The Pt shows high dispersion on the monolayer $\mathrm{MoS}_{2}$ as surfacebound single atoms and small nanocrystals. The enriched edges and defects of dendritic-shaped $\mathrm{MoS}_{2}$ show HER activities and are the preferential sites for stabilizing the Pt via chemical binding. These endow the Pt:MoS 2 dendrite with a greatly higher density of electrocatalytically active sites both at its edges and on its surface covering a large area up to sub-cm scale. In addition, the monolayer thickness and the dendritic morphology lead to rapid charge transfer and low resistance. The $\mathrm{Pt}$ and $\mathrm{MoS}_{2}$ not only engage in the catalysis individually, but also have synergistic effects, i.e., the Pt-S bonds improve the HER behavior of $\mathrm{Mo}$ at the edges and create more catalytic sites on the surface. The rate-limiting step in the HER is thereby regulated from Volmer towards 
Heyrovsky route, demonstrating the interactive impacts of $\mathrm{MoS}_{2}$ shape engineering and its hybridization with Pt on achieving increased hydrogen evolution efficiency. By demonstrating the direct synthesis of 2D nanocatalysts with reasonable performance, good conductivity, and high durability, we have paved a feasible avenue for practical application in water electrolysis.

\section{EXPERIMENTAL SECTION}

APCVD Synthesis of Pt:MoS 2 Hybrid. The Pt:MoS 2 hybrids were grown by APCVD using molybdenum trioxide $\left(\mathrm{MoO}_{3}\right.$, powder, $\geq 99.5 \%$, Sigma-Aldrich) and sulfur (S, powder, $\geq 99.5 \%$, Sigma-Aldrich) as the precursors. Chloroplatinic acid hydrate $\left(\mathrm{H}_{2} \mathrm{PtCl}_{6} \cdot x \mathrm{H}_{2} \mathrm{O}, 99.995 \%\right.$, SigmaAldrich) was the Pt source. The growth substrate, conductive GC thin plate $(2 \mathrm{~cm} \times 1.5 \mathrm{~cm})$, was sonicated for $10 \mathrm{~min}$ in acetone and isopropanol solution successively. The $\mathrm{H}_{2} \mathrm{PtCl}_{6} \cdot x \mathrm{H}_{2} \mathrm{O}$ was dehydrated at $220{ }^{\circ} \mathrm{C}$ under vacuum for $\sim 2 \mathrm{~h}$. The $\mathrm{PtCl}_{4}$ product was dissolved into acetone and dispersed by sonication to prepare $0.01 \mathrm{M}$ solution, which were then drop-casted onto the GC substrate surface through $\mu \mathrm{L}$ burette and carefully applied with cotton bud. We employed doublewalled quartz tubes for the synthesis to avoid cross-reaction between the reactants of $\mathrm{MoO}_{3}$ and $\mathrm{S}$. Two separate furnaces were utilized for individual temperature control. The $\mathrm{MoO}_{3}(15 \mathrm{mg}$ ) put in the inner tube (0.6 inch diameter) was positioned in the central zone of the low-temperature furnace, and the $\mathrm{S}(600 \mathrm{mg})$ put in the outer tube (1 inch diameter) was positioned $1.7 \mathrm{~cm}$ from the left open end of the high-temperature furnace. The GC plate was located downstream at the center of the high-temperature furnace (Figure 1b). To produce triangular domains, the GC substrate was placed near the medial axis of the outer tube, where the Mo:S ratio was $\sim 1: 2$ during the synthesis, resulting in thermodynamic growth. To produce dendritic domains, the GC substrate was placed in close proximity to the wall of the outer tube, where the Mo:S ratio was $<<1: 2$, deriving kinetic 
growth. ${ }^{36,71}$ After driving off the oxygen and moisture by flushing the reaction system with 500 sccm argon (Ar) for $30 \mathrm{~min}$, the low- and high-temperature furnaces were ramped up to $\sim 180$ and $\sim 200{ }^{\circ} \mathrm{C}$, respectively, whereby a sulfur-rich environment was created. The vaporous precursors were transported to the GC substrate surface by the carrier gases of argon (Ar) and hydrogen $\left(\mathrm{H}_{2}\right)$. (1) Nucleation. The high-temperature furnace was heated up to $800{ }^{\circ} \mathrm{C}$. The temperature of $\mathrm{MoO}_{3}$ was raised to $300{ }^{\circ} \mathrm{C}$ in the meanwhile. Once the target temperature was reached, the $\mathrm{MoS}_{2}$ began to nucleate with $150 \mathrm{sccm}$ Ar flow and $10 \mathrm{sccm} \mathrm{H}_{2}$ flow. Plenty of nuclei could be formed within 20 min. (2) Main growth. This stage lasted for $25 \mathrm{~min}$, with the Ar flow rate decreased to $50 \mathrm{sccm}$. (3) Edge attachment. We devised this 15 min process in order to get highly uniform monolayer structures and increase the domain size, which was realized by allowing the atoms to migrate freely across the surfaces of $\mathrm{GC}$ and $\mathrm{MoS}_{2}$ before attaching to the more thermodynamically favorable edges. The Ar and $\mathrm{H}_{2}$ flow rates were respectively dropped to 5 and $0 \mathrm{sccm}$ for lower precursor feedstocks. The GC temperature was decreased to $700{ }^{\circ} \mathrm{C}$ so as to attenuate the sublimation of $\mathrm{MoS}_{2}$ that had been synthesized on the substrate. The $\mathrm{MoO}_{3}$ temperature was reduced to $270{ }^{\circ} \mathrm{C}$, in which way the production of dendrites following a DLA-like process could be guaranteed by the declined Mo:S ratio. The temperature of S slowly climbed from $180{ }^{\circ} \mathrm{C}$ to $200{ }^{\circ} \mathrm{C}$ during (2) and (3) to maintain the $\mathrm{S}$ vapor concentration at a nearly constant level. (4) Thermal annealing. As soon as the synthesis was finished, we annealed the sample in situ at $350{ }^{\circ} \mathrm{C}$ for $25 \mathrm{~min}$, leading to enhanced physical adhesions of both the Pt:MoS 2 hybrid to the GC support. Finally, it ended up with fast cooling.

Characterization. The samples were imaged at room temperate utilizing optical microscope, scanning electron microscope (SEM, Hitachi-4300, $3.0 \mathrm{kV}$ accelerating voltage), and ADF-STEM (JEOL ARM-200F STEM) with a CEOS aberration corrector, $80 \mathrm{kV}$ accelerating voltage). Dwell 
times of $32 \mu \mathrm{S}$ and a pixel size of $0.0073-0.015 \mathrm{~nm} \mathrm{px}^{-1}$ were applied for the ADF-STEM imaging, along with a $30 \mu \mathrm{m}$ CL aperture, $24.6 \mathrm{mrad}$ convergence semiangle, $12 \mathrm{pA}$ beam current, and 39-156 mrad inner acquisition angle. The ADF-STEM images were subjected to a Gaussian smooth. The Raman and PL spectra were recorded with a JY Horiba LabRAM ARAMIS imaging confocal Raman microscope. The domain was excited by a $12.5 \mathrm{~mW}, 532 \mathrm{~nm}(2.33 \mathrm{eV})$ diode laser, which was focused down to a $\sim 1 \mu \mathrm{m}$ spot. The acquisition times for spectra and maps were 1 and $0.1 \mathrm{~s}$, respectively. AFM was used to measure the layer thickness by Asylum Research MFP3D in AC mode with a silicon AC-160TS cantilever (Olympus, spring constant of $\sim 42 \mathrm{~N} \mathrm{~m}^{-1}$ and resonant frequency of $\sim 300 \mathrm{kHz}$ ). As for the XPS, the samples were examined using a Thermo Scientific K-Alpha instrument equipped with a microfocused monochromated Al X-ray source, which was operated at $12 \mathrm{keV}$. The diameter of the spot was $400 \mu \mathrm{m}$ and the detection depth was $10 \mathrm{~nm}$. The analyzer operates at a constant analyzer energy (CAE) of $200 \mathrm{eV}$ for survey scans and $50 \mathrm{eV}$ for detailed scans. The charges were neutralized by a combined low energy/ion flood source. Data acquisition and analysis were performed with Thermo Scientifics Advantage and CasaXPS software, respectively.

Electrochemical Measurements. The catalytic performance was tested in a three-electrode system, using the Pt:MoS 2 or pristine $\mathrm{MoS}_{2}$ grown on the $\mathrm{GC}$ as the working electrode, a carbon rod as the counter electrode, and $\mathrm{Ag} / \mathrm{AgCl} / \mathrm{KCl}(3 \mathrm{M})$ as the reference electrode. A defined working area of the sample was immerged into a $0.5 \mathrm{M} \mathrm{H}_{2} \mathrm{SO}_{4} \mathrm{~N}_{2}$-purged solution for HER activities. The potentials were calibrated to a reversible hydrogen electrode, i.e., $\mathrm{E}(\mathrm{RHE})=\mathrm{E}(\mathrm{Ag} / \mathrm{AgCl} / \mathrm{KCl}(3$ M)) $+0.21-0.059 \mathrm{pH}$. The LSV was conducted under quasi-equilibrium conditions at $1 \mathrm{mV} \mathrm{s}^{-1}$, whereas the cyclic voltammetry (CV) was applied at a fast scan rate of $100 \mathrm{mV} \mathrm{s}^{-1}$. EIS was 
measured by sweeping the frequency from 200000 to $1 \mathrm{~Hz}$ with a perturbation voltage amplitude of $10 \mathrm{mV}$. The chronoamperometry was carried out at the overpotential of $-0.2 \mathrm{~V}$ vs RHE.

Supporting Information

Edges and grain boundaries of pristine $\mathrm{MoS}_{2}$ dendrites, Pt loading on monolayer $\mathrm{MoS}_{2}$ triangles, effects of heavy Pt loading on APCVD, elemental composition and chemical states in Pt:Mo alloys, ADF-STEM images of the Pt on monolayer $\mathrm{MoS}_{2}$, wide-range Tafel plots, individual and synergistic effects of $\mathrm{Pt}$ and $\mathrm{MoS}_{2}$ on the HER, XPS measurements of the degraded and stabilized Pt:MoS 2 dendrites, HER performances of the Pt-decorated $\mathrm{MoS}_{2}$ reported to date

\section{ACKNOWLEDGMENTS}

J.H.W. thanks The University of Texas at Austin for support.

\section{REFERENCES}

(1) Norskov, J. K.; Christensen, Claus H. Toward Efficient Hydrogen Production at Surfaces. Science 2006, 312, 1322-1323.

(2) Morales-Guio, C. G.; Stern, L.-A.; Hu, X. Nanostructured Hydrotreating Catalysts for Electrochemical Hydrogen Evolution. Chem. Soc. Rev. 2014, 43, 6555-6569.

(3) Benck J. D.; Hellstern, T. R.; Kibsgaard, J.; Chakthranont, P.; Jaramillo, T. F. Catalyzing the Hydrogen Evolution Reaction (HER) with Molybdenum Sulfide Nanomaterials. ACS Catal. 2014, 4, 3957-3971.

(4) Hinnemann, B.; Moses, P. G.; Bonde, J.; Jørgensen, K. P.; Nielsen, J. H.; Horch, S.; Chorkendorff, I.; Nørskov, J. K. Biomimetic Hydrogen Evolution: $\mathrm{MoS}_{2}$ Nanoparticles as 
Catalyst for Hydrogen Evolution. J. Am. Chem. Soc. 2005, 127, 5308-5309.

(5) Jaramillo, T. F.; Jørgensen, K. P.; Bonde, J.; Nielsen, J. H.; Horch, S.; Chorkendorff, I. Identification of Active Edge Sites for Electrochemical $\mathrm{H}_{2}$ Evolution from $\mathrm{MoS}_{2}$ Nanocatalysts. Science 2007, 317, 100-102.

(6) Yang, Y.; Fei, H.; Ruan, G.; Xiang, C.; Tour, J. M. Edge-Oriented MoS 2 Nanoporous Films as Flexible Electrodes for Hydrogen Evolution Reactions and Supercapacitor Devices. $A d v$. Mater. 2014, 26, 8163-8168.

(7) Li, S.; Lee, J. K.; Zhou, S.; Pasta, M.; Warner, J. H. Synthesis of Surface Grown Pt Nanoparticles on Edge-Enriched $\mathrm{MoS}_{2}$ Porous Thin Films for Enhancing Electrochemical Performance. Chem. Mater. 2019, 31, 387-397.

(8) Kong, D.; Wang, H.; Cha, J. J.; Pasta, M.; Koski, K. J.; Yao, J.; Cui, Y. Synthesis of $\mathrm{MoS}_{2}$ and $\mathrm{MoSe}_{2}$ Films with Vertically Aligned Layers. Nano Lett. 2013, 13, 1341-1347.

(9) Li, S.; Wang, S.; Salamone, M. M.; Robertson, A. W.; Nayak, S.; Kim, H.; Tsang, S. C. E.; Pasta, M.; Warner, J. H. Edge-Enriched 2D $\mathrm{MoS}_{2}$ Thin Films Grown by Chemical Vapor Deposition for Enhanced Catalytic Performance. ACS Catal. 2017, 7, 877-886.

(10) Xu, W.; Li, S.; Zhou, S.; Lee, J. K.; Wang, S.; Sarwat, S. G.; Wang, X.; Bhaskaran, H.; Pasta, M.; Warner, J. H. Large Dendritic Monolayer $\mathrm{MoS}_{2}$ Grown by Atmospheric Pressure Chemical Vapor Deposition for Electrocatalysis. ACS Appl. Mater. Interfaces 2018, 10, $4630-4639$.

(11) Sun, Y.; Alimohammadi, F.; Zhang, D.; Guo, G. Enabling Colloidal Synthesis of EdgeOriented $\mathrm{MoS}_{2}$ with Expanded Interlayer Spacing for Enhanced HER Catalysis. Nano Lett. 2017, 17, 1963-1969.

(12) Lukowski, M. A.; Daniel, A. S.; Meng, F.; Forticaux, A.; Li, L.; Jin, S. Enhanced Hydrogen 
Evolution Catalysis from Chemically Exfoliated Metallic $\mathrm{MoS}_{2}$ Nanosheets. J. Am. Chem. Soc. 2013, 135, 10274-10277.

(13) Voiry, D.; Salehi, M.; Silva, R.; Fujita, T.; Chen, M.; Asefa, T.; Shenoy, V. B.; Eda, G.; Chhowalla, M. Conducting $\mathrm{MoS}_{2}$ Nanosheets as Catalysts for Hydrogen Evolution Reaction. Nano Lett. 2013, 13, 6222-6227.

(14) Yin, Y.; Han, J.; Zhang, Y.; Zhang, X.; Xu, P.; Yuan, Q.; Samad, L.; Wang, X.; Wang, Y.; Zhang, Z.; Zhang, P.; Cao, X.; Song, B.; Jin, S. Contributions of Phase, Sulfur Vacancies, and Edges to the Hydrogen Evolution Reaction Catalytic Activity of Porous Molybdenum Disulfide Nanosheets. J. Am. Chem. Soc. 2016, 138, 7965-7972.

(15) Li, H.; Tsai, C.; Koh, A. L.; Cai, L.; Contryman, A. W.; Fragapane, A. H.; Zhao, J.; Han, H. S.; Manoharan, H. C.; Abild-Pedersen, F.; Nørskov, J. K.; Zheng, X. Activating and Optimizing $\mathrm{MoS}_{2}$ Basal Planes for Hydrogen Evolution through the Formation of Strained Sulphur Vacancies. Nat. Mater. 2016, 15, 48-53.

(16) Ye, G.; Gong, Y.; Lin, J.; Li, B.; He, Y.; Pantelides, S. T.; Zhou, W.; Vajtai, R.; Ajayan, P. M. Defects Engineered Monolayer $\mathrm{MoS}_{2}$ for Improved Hydrogen Evolution Reaction. Nano Lett. 2016, 16, 1097-1103.

(17) Huang, N.; Peng, R.; Ding, Y.; Yan, S.; Li, G.; Sun, P.; Sun, X.; Liu, X.; Yu, H. Facile Chemical-Vapour-Deposition Synthesis of Vertically Aligned Co-Doped $\mathrm{MoS}_{2}$ Nanosheets as an Efficient Catalyst for Triiodide Reduction and Hydrogen Evolution Reaction. J. Catal. 2019, 373, 250-259.

(18) Wang, Y.; Udyavara, S.; Neurock, M.; Frisbie, C. D. Field Effect Modulation of Electrocatalytic Hydrogen Evolution at Back-Gated Two-Dimensional MoS2 Electrodes. Nano Lett. 2019, 19, 6118-6123. 
(19) Li, G.; Chen, Z.; Li, Y.; Zhang, D.; Yang, W.; Liu, Y.; Cao, L. Engineering Substrate Interaction to Improve Hydrogen Evolution Catalysis of Monolayer $\mathrm{MoS}_{2}$ Films Beyond Pt. ACS Nano 2020, 14, 1707-1714.

(20) Zheng, X.; Xu, J.; Yan, K.; Wang, H.; Wang, Z.; Yang, S. Space-Confined Growth of MoS2 Nanosheets within Graphite: The Layered Hybrid of $\mathrm{MoS}_{2}$ and Graphene as an Active Catalyst for Hydrogen Evolution Reaction. Chem. Mater. 2014, 26, 2344-2353.

(21) Gao, M.-R.; Liang, J.-X.; Zheng, Y.-R.; Xu, Y.-F.; Jiang, J.; Gao, Q.; Li, J.; Yu, S.-H. An Efficient Molybdenum Disulfide/Cobalt Diselenide Hybrid Catalyst for Electrochemical Hydrogen Generation. Nat. Commun. 2015, 6, 5982.

Zuo, P.; Jiang, L.; Li, X.; Li, B.; Ran, P.; Li, X.; Qu, L.; Lu, Y. Metal (Ag, Pt)-MoS 2 Hybrids Greenly Prepared Through Photochemical Reduction of Femtosecond Laser Pulses for SERS and HER. ACS Sustainable Chem. Eng. 2018, 6, 7704-7714.

(23) Xu, Q.; Liu, Y.; Jiang, H.; Hu, Y.; Liu, H.; Li, C. Unsaturated Sulfur Edge Engineering of Strongly Coupled $\mathrm{MoS}_{2}$ Nanosheet-Carbon Macroporous Hybrid Catalyst for Enhanced Hydrogen Generation. Adv. Energy Mater. 2019, 9, 1802553.

(24) Zhou, Y.; Pondick, J. V.; Silva, J. L.; Woods, J. M.; Hynek, D. J.; Matthews, G.; Shen, X.; Feng, Q.; Liu, W.; Lu, Z.; Liang, Z.; Brena, B.; Cai, Z.; Wu, M.; Jiao, L.; Hu, S.; Wang, H.; Araujo, C. M.; Cha, J. J. Unveiling the Interfacial Effects for Enhanced Hydrogen Evolution Reaction on $\mathrm{MoS}_{2} / \mathrm{WTe}_{2}$ Hybrid Structures. Small, 2019, 15, 1900078.

Li, G.; Zhang, D.; Qiao, Q.; Yu, Y.; Peterson, D.; Zafar, A.; Kumar, R.; Curtarolo, S.; Hunte, F.; Shannon, S.; Zhu, Y.; Yang, W.; Cao, L. All the Catalytic Active Sites of $\mathrm{MoS}_{2}$ for Hydrogen Evolution. J. Am. Chem. Soc. 2016, 138, 16632-16638.

(26) Deng, J.; Li, H.; Xiao, J.; Tu, Y.; Deng, D.; Yang, H.; Tian, H.; Li, J.; Ren, P.; Bao, X. 
Triggering the Electrocatalytic Hydrogen Evolution Activity of the Inert Two-Dimensional $\mathrm{MoS}_{2}$ Surface via Single-Atom Metal Doping. Energy Environ. Sci. 2015, 8, 1594-1601.

(27) Yuwen, L.; Xu, F.; Xue, B.; Luo, Z.; Zhang, Q.; Bao, B.; Su, S.; Weng, L.; Huang, W.; Wang, L. General Synthesis of Noble Metal (Au, Ag, Pd, Pt) Nanocrystal Modified $\mathrm{MoS}_{2}$ Nanosheets and the Enhanced Catalytic Activity of $\mathrm{Pd}-\mathrm{MoS}_{2}$ for Methanol Oxidation. Nanoscale 2014, 6, 5762-5769.

(28) Ying, J.; Jiang, G.; Paul Cano, Z.; Han, L.; Yang, X. Y.; Chen, Z. Nitrogen-Doped Hollow Porous Carbon Polyhedrons Embedded with Highly Dispersed Pt Nanoparticles as a Highly Efficient and Stable Hydrogen Evolution Electrocatalyst. Nano Energy 2017, 40, 88-94.

(29) Deng, D.; Novoselov, K. S.; Fu, Q.; Zheng, N.; Tian, Z.; Bao, X. Catalysis with TwoDimensional Materials and Their Heterostructures. Nat. Nanotechnol. 2016, 11, 218-230.

(30) Li, H.; Wang, S.; Sawada, H.; Han, G. G. D.; Samuels, T.; Allen, C. S.; Kirkland, A. I.; Grossman, J. C.; Warner, J. H. Atomic Structure and Dynamics of Single Platinum Atom Interactions with Monolayer $\mathrm{MoS}_{2}$. ACS Nano 2017, 11, 3392-3403.

Li, H.; Du, M.; Mleczko, M. J.; Koh, A. L.; Nishi, Y.; Pop, E.; Bard, A. J.; Zheng, X. Kinetic Study of Hydrogen Evolution Reaction over Strained $\mathrm{MoS}_{2}$ with Sulfur Vacancies Using Scanning Electrochemical Microscopy. J. Am. Chem. Soc. 2016, 138, 5123-5129.

(32) Xie, J.; Gao, L.; Jiang, H.; Zhang, X.; Lei, F.; Hao, P.; Tang, B.; Xie, Y. Platinum Nanocrystals Decorated on Defect-Rich $\mathrm{MoS}_{2}$ Nanosheets for $\mathrm{pH}$-Universal Hydrogen Evolution Reaction. Cryst. Growth Des. 2019, 19, 60-65.

(33) Wang, S.; Sawada, H.; Han, X.; Zhou, S.; Li, S.; Guo, Z. X.; Kirkland, A. I.; Warner, J. H. Preferential Pt Nanocluster Seeding at Grain Boundary Dislocations in Polycrystalline Monolayer MoS 2 . ACS Nano 2018, 12, 5626-5636. 
(34) Zhang, Y.; Ji, Q.; Wen, J.; Li, J.; Li, C.; Shi, J.; Zhou, X.; Shi, K.; Chen, H.; Li, Y.; Deng, S.; Xu, N.; Liu, Z.; Zhang, Y. Monolayer $\mathrm{MoS}_{2}$ Dendrites on a Symmetry-Disparate $\mathrm{SrTiO}_{3}$ (001) Substrate: Formation Mechanism and Interface Interaction. Adv. Funct. Mater. 2016, 26, 3299-3305.

(35) Yu, Y.; Huang, S.-Y.; Li, Y.; Steinmann, S. N.; Yang, W.; Cao, L. Layer-Dependent Electrocatalysis of $\mathrm{MoS}_{2}$ for Hydrogen Evolution. Nano Lett. 2014, 14, 553-558.

(36) Wang, J.; Cai, X.; Shi, R.; Wu, Z.; Wang, W.; Long, G.; Tang, Y.; Cai, N.; Ouyang, W.; Geng, P.; Chandrashekar, B. N.; Amini, A.; Wang, N.; Cheng, C. Twin Defect Derived Growth of Atomically Thin $\mathrm{MoS}_{2}$ Dendrites. ACS Nano 2017, 12, 635-643.

Zhang, K.; Feng, S.; Wang, J.; Azcatl, A.; Lu, N.; Addou, R.; Wang, N.; Zhou, C.; Lerach, J.; Bojan, V.; Kim, M. J.; Chen, L.-Q.; Wallace, R. M.; Terrones, M.; Zhu, J.; Robinson, J. A. Manganese Doping of Monolayer $\mathrm{MoS}_{2}$ : The Substrate Is Critical. Nano Lett. 2015, 15, $6586-6591$.

(38) Splendiani, A.; Sun, L.; Zhang, Y.; Li, T.; Kim, J.; Chim, C.-Y.; Galli, G.; Wang, F. Emerging Photoluminescence in Monolayer $\mathrm{MoS}_{2}$. Nano Lett. 2010, 10, 1271-1275.

(39) Stojić, D. L.; Grozdić, T. D.; Kaninski, M. P. M.; Stanić, V. Đ. Electrocatalytic Effects of Mo-Pt Intermetallics Singly and with Ionic Activators. Hydrogen Production via Electrolysis. Int. J. Hydrogen Energy 2007, 32, 2314-2319.

(40) Ren, W.; Zhang, H.; Cheng, C. Ultrafine Pt Nanoparticles Decorated $\mathrm{MoS}_{2}$ Nanosheets with Significantly Improved Hydrogen Evolution Activity. Electrochim. Acta 2017, 241, 316322.

(41) Chen, P.; Xu, W.; Gao, Y.; Holdway, P.; Warner, J. H.; Castell, M. R. Thermal Degradation of Monolayer $\mathrm{MoS}_{2}$ on $\mathrm{SrTiO}_{3}$ Supports. J. Phys. Chem. C 2019, 123, 3876-3885. 
(42) Hou, D.; Zhou, W.; Liu, X.; Zhou, K.; Xie, J.; Li, G.; Chen, S. Pt Nanoparticles/MoS2 Nanosheets/Carbon Fibers as Efficient Catalyst for the Hydrogen Evolution Reaction. Electrochim. Acta 2015, 166, 26-31.

(43) Wu, C.; Li, D.; Ding, S.; Rehman, Z. U.; Liu, Q.; Chen, S.; Zhang, B.; Song, Li. Monoatomic Platinum-Anchored Metallic $\mathrm{MoS}_{2}$ : Correlation between Surface Dopant and Hydrogen Evolution. J. Phys. Chem. Lett. 2019, 10, 6081-6087.

(44) Saidi, W. A. Density Functional Theory Study of Nucleation and Growth of Pt Nanoparticles on $\mathrm{MoS}_{2}(001)$ Surface. Cryst. Growth Des. 2015, 15, 642-652.

(45) Saidi, W. A. Trends in the Adsorption and Growth Morphology of Metals on the $\mathrm{MoS}_{2}(001)$ Surface. Cryst. Growth Des. 2015, 15, 3190-3200.

(46) Shi, Y.; Song, B.; Shahbazian-Yassar, R.; Zhao, J.; Saidi, W. A. Experimentally Validated Structures of Supported Metal Nanoclusters on $\mathrm{MoS}_{2}$. J. Phys. Chem. Lett. 2018, 9, 2972-2978.

(47) Gao, J.; Zou, J.; Zeng, X.; Ding, W. Carbon Supported Nano Pt-Mo Alloy Catalysts for Oxygen Reduction in Magnesium-Air Batteries. RSC Adv. 2016, 6, 83025-83030.

(48) Wang, J.; Cao, X.; Fang, L.; You, X.; Wong, K.; Cao, S.; Xiao, C.; Cai, S.; Huang, Y.; Zhang, X.; Chen, Z. $\mathrm{MoS}_{2}$ Nanoflower Supported Pt Nanoparticle as an Efficient Electrocatalyst for Ethanol Oxidation Reaction. Int. J. Hydrogen Energy 2019, 44, 16411-16423.

(49) Vračar, L.; Krstajić, N.; Neophytides, S. G.; Jakšić, J. Underpotential Deposition of Hydrogen on $\mathrm{MoPt}_{4}$ Intermetallic Phase in Acid Solution: Temperature Dependence. Int. J. Hydrogen Energy 2004, 29, 835-842.

(50) Zosimova, P. A.; Smirnov, A. V.; Nesterenko, S. N.; Yuschenko, V. V.; Sinkler, W.; Kocal, J.; Holmgren, J.; Ivanova, I. I. Synthesis, Characterization, and Sulfur Tolerance of 
Pt- $\mathrm{MoO}_{x}$ Catalysts Prepared from Pt-Mo Alloy Precursors. J. Phys. Chem. C 2007, 111, $14790-14798$.

(51) Wang, S.; Sawada, H.; Chen, Q.; Han, G. G. D.; Allen, C.; Kirkland, A. I.; Warner, J. H. In Situ Atomic-Scale Studies of the Formation of Epitaxial Pt Nanocrystals on Monolayer Molybdenum Disulfide. ACS Nano 2017, 11, 9057-9067.

(52) Ferreira, P. J.; la O’, G. J.; Shao-Horn, Y.; Morgan, D.; Makharia, R.; Kocha, S.; Gasteiger, H. A. Instability of Pt/C Electrocatalysts in Proton Exchange Membrane Fuel Cells: A Mechanistic Investigation. J. Electrochem. Soc. 2005, 152, A2256-A2271.

(53) Lee, C.; Yan, H.; Brus, L. E.; Heinz, T. F.; Hone, J.; Ryu, S. Anomalous Lattice Vibrations of Single- and Few-Layer MoS 2 . ACS Nano 2010, 4, 2695-2700.

(54) Rice, C.; Young, R. J.; Zan, R.; Bangert, U.; Wolverson, D.; Georgiou, T.; Jalil, R.; Novoselov, K. S. Raman-Scattering Measurements and First-Principles Calculations of Strain-Induced Phonon Shifts in Monolayer MoS 2 . Phys. Rev. B 2013, 87, 081307.

(55) Chakraborty, B.; Bera, A.; Muthu, D. V. S.; Bhowmick, S.; Waghmare, U. V.; Sood, A. K. Symmetry-Dependent Phonon Renormalization in Monolayer MoS2 Transistor. Phys. Rev. $B$ 2012, 85, 161403 .

Qiu, H.; Xu, T.; Wang, Z.; Ren, W.; Nan, H.; Ni, Z.; Chen, Q.; Yuan, S.; Miao, F.; Song, F.; Long, G.; Shi, Y.; Sun, L.; Wang, J.; Wang, X. Hopping Transport through Defect-Induced Localized States in Molybdenum Disulphide. Nat. Commun. 2013, 4, 2642.

(57) Cheng, Y. C.; Zhang, Q. Y.; Schwingenschlögl, U. Valley Polarization in Magnetically Doped Single-Layer Transition-Metal Dichalcogenides. Phys. Rev. B 2014, 89, 155429.

(58) Wang, S.; Wang, X.; Warner, J. H. All Chemical Vapor Deposition Growth of MoS2:h-BN Vertical van der Waals Heterostructures. ACS Nano 2015, 9, 5246-5254. 
Xu, W.; Kozawa, D.; Liu, Y.; Sheng, Y.; Wei, K.; Koman, V. B.; Wang, S.; Wang, X.; Jiang, T.; Strano, M. S.; Warner, J. H. Determining the Optimized Interlayer Separation Distance in Vertical Stacked 2D WS $2: \mathrm{hBN}: \mathrm{MoS}_{2}$ Heterostructures for Exciton Energy Transfer. Small 2018, $14,1703727$.

(60) Nan, H.; Wang, Z.; Wang, W.; Liang, Z.; Lu, Y.; Chen, Q.; He, D.; Tan, P.; Miao, F.; Wang, X.; Wang, J.; Ni, Z. Strong Photoluminescence Enhancement of $\mathrm{MoS}_{2}$ through Defect Engineering and Oxygen Bonding. ACS Nano 2014, 8, 5738-5745.

(61) Tiwari, J. N.; Sultan, S.; Myung, C. W.; Yoon, T.; Li, N.; Ha, M.; Harzandi, A. M.; Park, H. J.; Kim, D. Y.; Chandrasekaran, S. S.; Lee, W. G.; Vij, V.; Kang, H.; Shin, T. J.; Shin, H. S.; Lee, G.; Lee, Z.; Kim, K. S. Multicomponent Electrocatalyst with Ultralow Pt Loading and High Hydrogen Evolution Activity. Nat. Energy 2018, 3, 773-782.

(62) Jagminas, A.; Naujokaitis, A.; Žalnèravičius, R.; Jasulaitiene, V.; Valušis, G. Tuning the Activity of Nanoplatelet $\mathrm{MoS}_{2}$-Based Catalyst for Efficient Hydrogen Evolution via Electrochemical Decoration with Pt Nanoparticles. Appl. Surf. Sci. 2016, 385, 56-62.

(63) Jagminas, A.; Naujokaitis, A.; Žalnėravičius, R.; Jasulaitiene, V.; Valušis, G. Tuning the Activity of Nanoplatelet $\mathrm{MoS}_{2}$-Based Catalyst for Efficient Hydrogen Evolution via Electrochemical Decoration with Pt Nanoparticles. Appl. Surf. Sci. 2016, 385, 56-62.

(64) Lazar, P.; Otyepka, M. Role of the Edge Properties in the Hydrogen Evolution Reaction on $\mathrm{MoS}_{2}$. Chem. Eur. J. 2017, 23, 4863-4869.

Li, L.; Qin, Z.; Ries, L.; Hong, S.; Michel, T.; Yang, J.; Salameh, C.; Bechelany, M.; Miele, P.; Kaplan, D.; Chhowalla, M.; Voiry, D. Role of Sulfur Vacancies and Undercoordinated Mo Regions in $\mathrm{MoS}_{2}$ Nanosheets toward the Evolution of Hydrogen. ACS Nano 2019, 13, $6824-6834$. 
(66) Yang, T. T.; Tan, T. L.; Saidi, W. A. High Activity toward the Hydrogen Evolution Reaction on the Edges of $\mathrm{MoS}_{2}$-Supported Platinum Nanoclusters Using Cluster Expansion and Electrochemical Modeling. Chem. Mater. 2020, 32, 1315-1321.

(67) Trasatti, S. Work Function, Electronegativity, and Electrochemical Behaviour of Metals: III. Electrolytic Hydrogen Evolution in Acid Solutions. J. Electroanal. Chem. Interfacial Electrochem. 1972, 39, 163-184.

(68) Paciok, P.; Schalenbach, M.; Carmo, M.; Stolten, D. On the Mobility of Carbon-Supported Platinum Nanoparticles towards Unveiling Cathode Degradation in Water Electrolysis. $J$. Power Sources 2017, 365, 53-60.

(69) Murthy, A. P.; Theerthagiri, J.; Madhavan, J.; Murugan, K. Highly Active $\mathrm{MoS}_{2} /$ Carbon Electrocatalysts for the Hydrogen Evolution Reaction - Insight into the Effect of the Internal Resistance and Roughness Factor on the Tafel Slope. Phys. Chem. Chem. Phys. 2017, 19, 1988-1998.

(70) Nagasubramanian, G. Two- and Three-Electrode Impedance Studies on 18650 Li-Ion Cells. J. Power Sources 2000, 87, 226-229.

(71) Xu, W.; Kozawa, D.; Zhou, Y.; Wang, Y.; Sheng, Y.; Jiang, T.; Strano, M. S.; Warner, J. H. Controlling Photoluminescence Enhancement and Energy Transfer in $\mathrm{WS}_{2}: \mathrm{hBN}: \mathrm{WS}_{2}$ Vertical Stacks by Precise Interlayer Distances. Small 2019, 16, 1905985.

(72) Tsai, C.; Abild-Pedersen, F.; Nørskov, J. K. Tuning the $\mathrm{MoS}_{2}$ Edge-Site Activity for Hydrogen Evolution via Support Interactions. Nano Lett. 2014, 14, 1381-1387.

(73) Wang, S.; Rong, Y.; Fan, Y.; Pacios, M.; Bhaskaran, H.; He, K.; Warner, J. H. Shape Evolution of Monolayer $\mathrm{MoS}_{2}$ Crystals Grown by Chemical Vapor Deposition. Chem. Mater. 2014, 26, 6371-6379. 
ToC Graphic

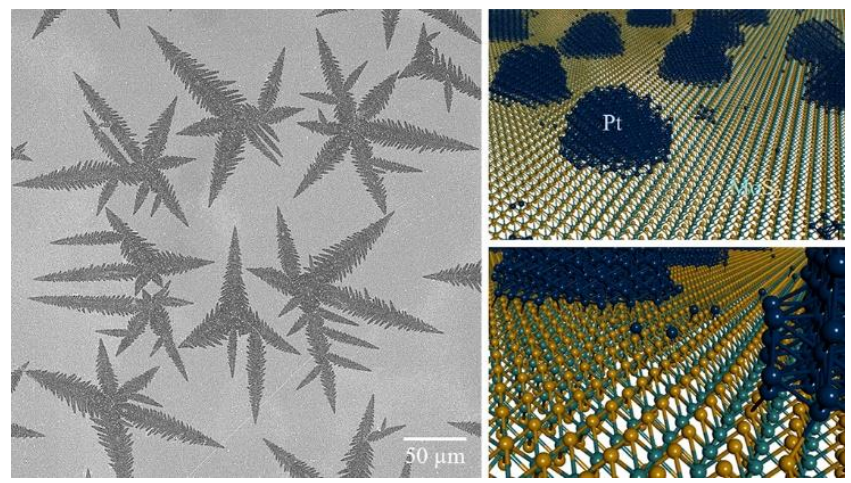

